\title{
Propriedades ópticas de filmes finos de MEH-PPV preparados por "spin-coating" em diferentes velocidades de rotação
}

\section{Optical properties of thin films of MEH-PPV produced by the spin- coating technique at different rotational speeds}

\author{
Marco Aurélio Toledo da Silva ${ }^{1}$; Flávio Alexandre Costa de Oliveira ${ }^{2}$; Ivan \\ Frederico Lupiano Dias ${ }^{3}$; José Leonil Duarte ${ }^{4}$; Edson Laureto ${ }^{4}$; Sidney Alves \\ Lourenço $^{5}$; Luís Alberto Cury ${ }^{6}$; Paulo Sérgio Soares Guimarães ${ }^{6}$
}

Resumo

Apresentamos um estudo das propriedades ópticas de filmes finos de poly[2-methoxy-5-(20-ethylhexyloxy)-1,4-phenylene vinylene] (MEH-PPV), preparados em uma concentração de $10 \mathrm{mg} / \mathrm{ml}$ de xileno. A solução foi depositada sobre substrato de vidro por "spin-coating" em três rotações diferentes $(300,1000$ e $4000 \mathrm{rpm})$. Estudamos os efeitos da velocidade de rotação nas amostras a $300 \mathrm{~K}$, analisando os espectros de fotoluminescência (PL) em diferentes pontos do filme polimérico. Analisamos também os efeitos da intensidade de excitação no comportamento óptico do MEH-PPV a $300 \mathrm{~K}$. Em baixas, temperaturas os espectros de PL da amostra A1000 (1000 rpm) mostraram um pico estreito para transição puramente eletrônica e uma série de bandas vibrônicas que revelam o acoplamento com diferentes modos vibrônicos. Na amostra com rotação intermediária (1000 rpm) fizemos uma análise da emissão em função da variação da temperatura no intervalo de $130 \mathrm{~K}$ a $290 \mathrm{~K}$. Também analisamos de modo sistemático, a forma de linha por meio de gaussianas comparando as com a literatura.

Palavras-chave: Polímeros conjugados. Propriedades ópticas. MEH-PPV.

\begin{abstract}
We present a study on the optical properties of thin films of poly[2-methoxy-5-(20-ethyl-hexyloxy)1,4-phenylene vinylene] (MEH-PPV) produced at a concentration of $10 \mathrm{mg} / \mathrm{ml}$ xylene. The solution was deposited onto glass substrates by the spin-coating technique at different rotational speeds ( 300 , 1000 and $4000 \mathrm{rpm}$ ). We study the effect of rotational speeds on the sample at $300 \mathrm{~K}$, by analyzing the photoluminescence (PL) spectra at different points of the polymeric film. We also analyze the effects of the excitation power on the optical behavior of MEH-PPV at $300 \mathrm{~K}$. At low temperatures the PL spectra of sample A1000 (1000 rpm) show a narrow peak for the electronic transition and a series of vibronic sidebands which reveal the electron coupling with two different vibronic modes. In the temperature range of $130 \mathrm{~K}$ to $290 \mathrm{~K}$, we analyze systematically the transition lineshapes in the optical spectra using Gaussian curves.
\end{abstract}

Key words: Conjugated polymers. Optical properties. MEH-PPV.

\footnotetext{
1 Graduado e Mestre em Física, Universidade Estadual de Londrina. E-mail: seaquinhos@hotmail.com.

2 Graduado e Mestre em Física, Universidade Federal de Minas Gerais.

3 Docente do Departamento de Física da Universidade Estadual de Londrina - DFIS-UEL. E-mail: idias@uel.br.

4 Docente do Departamento de Física da Universidade Estadual de Londrina - DFIs-UEL.

5 Docente do Departamento de Física da Universidade Estadual Centro-Oeste.

6 Docente do Departamento de Física da Universidade Federal de Minas Gerais.
} 


\section{Introdução}

Polímero (do grego: poli $=$ muitos; mero $=$ unidade de repetição) é uma macromolécula composta por muitas unidades de repetição unidas por ligações primárias geralmente do tipo covalente (CANEVAROLO JÚNIOR, 2002).

Apesar polímeros serem conhecidos desde a antiguidade, a ciência dos polímeros em base molecular desenvolveu-se principalmente no século XX (HEEGER, 2001) com a contribuição de diversos cientistas, entre os quais citamos Hermann Staudinger, Karl Ziegler, Giulio Natta e Paul Flory. Todos receberam Prêmios Nobel em Química de 1953, Staudinger, pela concepção e desenvolvimento do conceito de macromoléculas, em 1963 Ziegler e Natta, pela síntese de polímeros relevantes para a indústria do plástico, e em 1974, Flory, por sua contribuição teórica e experimental ao estudo da físico-química das macromoléculas. Estes pesquisadores lançaram as bases da moderna ciência dos polímeros.

Os polímeros estudados por estes pesquisadores, denominados polímeros saturados, eram isolantes o que os tornava desinteressantes para a aplicação em dispositivos eletrônicos. Em 1977, conseguiu-se fabricar o primeiro polímero semicondutor, como o poliacetileno- $(\mathrm{CH})_{\mathrm{n}}$, e desenvolveu-se a tecnologia de controle de sua dopagem em um intervalo de concentração de dopantes que permitia variar o seu comportamento da condição de isolante até metálico (CHIANG et al., 1977; SHIRAKAWA et al., 1977). O Prêmio Nobel de Química de 2000 foi conferido a Alan J. Heeger, Alan MacDiarmid e Hideki Shirakawa pela descoberta e desenvolvimento de polímeros condutores. A estrutura eletrônica dos polímeros conjugados em termos de estrutura de bandas foi descrita por Su, Schrieffer e Heeger, utilizando um modelo "tight-binding" quase unidimensional ao final de década de 70 (SU; SCHRIEFFER; HEEGER, 1979, 1980).

A principal diferença entre os polímeros saturados e os conjugados está na sua configuração eletrônica.
Nos polímeros saturados, os quatro elétrons de valência do átomo de carbono $\left(1 \mathrm{~s}^{2} 2 \mathrm{~s}^{2} 1 \mathrm{p}_{\mathrm{x}}{ }^{1} 1 \mathrm{p}_{\mathrm{y}}{ }^{1}\right)$ sofrem um processo de hibridização com a formação de orbitais do tipo $\mathrm{sp}^{3}$ que são usados em ligações covalentes com outros quatro átomos formando ligações do tipo $\sigma$. Nos polímeros conjugados, os quatro elétrons de valência do carbono sofrem um processo de hibridização com a formação de orbitais do tipo $\mathrm{sp}^{2} \mathrm{e}$, conseqüentemente cada átomo de carbono se liga a três outros átomos. Assim, três dos quatro átomos do carbono são localizados em orbitais do tipo $\sigma$. O quarto elétron de valência permanece em um orbital do tipo $\mathrm{p}_{\mathrm{z}}$ possibilitando a formação da ligação do tipo $\pi$. A ligação $\pi$ caracteriza um estado mais delocalizado que forma orbitais moleculares e bandas $\pi$. As bandas $\pi$ darão um caráter metálico ou semicondutor ao polímero conjugado dependendo se estão ou não totalmente preenchidas. No poliacetileno, a banda $\pi$ é desdobrada em duas sub-bandas, uma banda $\pi$ completamente ocupada (a banda de valência) e uma sub-banda $\pi^{*}$ completamente desocupada (a banda de condução). Um dos aspectos interessantes e motivadores do estudo científico deste material, por exemplo, é o transporte por meio de sólitons (KIVELSON; SHRIEFFLER; HEEGER, 1988). O poliacetileno, entretanto não tem propriedades eletrônicas interessantes para aplicação em óptica uma vez que não luminesce (CÔTÉ, 2003).

Um polímero semicondutor luminescente de grande interesse é o PPV (poli - fenileno vinileno). Propriedades eletroluminescentes de polímeros foram registradas pela primeira vez usando o PPV como um material semicondutor entre eletrodos metálicos (BURROUGHES et al., 1990). No PPV - uma estrutura que consiste de uma cadeia de anéis benzênicos e grupos vinis, repetidas alternadamente - temos oito elétrons em cada unidade de repetição e a banda $\pi$ se desdobra em oito sub-bandas separadas por um intervalo de energia proibida. Cada sub-banda pode ser ocupada por dois elétrons ("spin up" e "spin down"). Assim, as quatro subbandas de menor energia estarão completamente 
preenchidas pelos oito elétrons provenientes dos orbitais $\mathrm{p}_{\mathrm{z}}$ de cada átomo de carbono da unidade de repetição da estrutura polimérica. Com uma subbanda completamente preenchida, a outra sub-banda completamente vazia e um intervalo de energia proibida ou "gap" $\left(\mathrm{E}_{\pi-\pi^{*}}\right)$ de aproximadamente 2.5 eV. O PPV pode ser caracterizado como um material semicondutor (CURY, 2004). Devido às suas propriedades luminescentes, compostos baseados no polímero conjugado PPV têm sido empregados para a fabricação de lasers (TESSLER; DENTON; FRIEND, 1996). Brazovskii, Kirova e Bishop (1998) e Kirova, Brazovskii e Bishop (1999) calcularam a estrutura eletrônica do PPV em termos de estrutura de bandas com uma aproximação do tipo "tightbinding". Trabalhos experimentais de absorção em PPV orientado e PPP (poli-parafenileno) mostraram que esta é uma boa aproximação (MILLER et al., 1999; MILLER; YANG; HEEGER, 2000).

De modo geral os polímeros conjugados absorvem fótons no intervalo de comprimento de onda do violeta ao azul (300 $\mathrm{nm}<\lambda<500 \mathrm{~nm})$ e emitem fótons no intervalo do verde ao vermelho (500 $\mathrm{nm}<\lambda<700 \mathrm{~nm})$. Este grande "Stokes shift" (o intervalo de energia entre a região de absorção e a de emissão) é uma das características marcantes dos polímeros conjugados e é muito maior que o "Stokes shift" existente em materiais semicondutores inorgânicos (CURY, 2004). Uma outra característica importante é a grande eficiência da emissão nos polímeros conjugados. O efeito da relaxação de elétrons via emissão de fônons é muito maior que em materiais semicondutores inorgânicos tais como o GaAs, o InP, etc. O processo de relaxação é extremamente rápido ocorrendo em períodos de tempo que podem variar de 100 fs a 1 ps, enquanto nos materiais semicondutores inorgânicos o intervalo de tempo característico é de 1 ps a 1 ns. Os picos de fotoluminescência dos polímeros conjugados (decaimento de éxciton com emissão de fônons) são muito alargados, quando comparados aos semicondutores inorgânicos, devido à grande interação elétron-fônon e ao espalhamento da luz emitida causado por imperfeições e/ou desordem da rede polimérica. Uma característica do espectro de emissão é, portanto, a presença de réplicas de fônons, devido a relaxação de elétrons em estados excitados para os vários níveis de energia associados às vibrações existentes na molécula polimérica. Os centros de aniquilação, por exemplo, os defeitos carbonílicos, podem reduzir drasticamente a eficiência da emissão, pois os elétrons podem se recombinar de forma não radiativa com estes defeitos. A formação destes centros em polímeros, de modo geral, entretanto, não é ainda bem compreendida.

A energia do fóton de luz emitido depende de como os orbitais $\mathrm{p}_{\mathrm{z}}$, os orbitais responsáveis pela criação da estrutura de bandas, interagem dentro do monômero. Pode-se controlar a altura da banda proibida modificando-se a estrutura química do material. No caso do PPV (que emite luz na região do verde, com $\mathrm{E}_{\pi-\pi^{*}} \sim 2,5 \mathrm{eV}$ ), pode-se promover alterações introduzindo radicais laterais à cadeia principal. No poly[2-methoxy-5-(2'-ethylhexoxy)p-phenylene vinylene] (MEH-PPV), um derivado do PPV, um grupo alcóxico $\left(\mathrm{OC}_{6} \mathrm{H}_{3}\right)$ introduzido lateralmente faz com que o "gap" diminua levando a emissões na região do laranja ao vermelho. $\mathrm{Na}$ Figura 1, apresentamos alguns polímeros conjugados de interesse tecnológico. O completo entendimento do comportamento da variação da banda de energia proibida depende de cálculos complexos e constitui um tema de pesquisa atual. Qualitativamente entende-se a variação do "gap" em termos da alteração na delocalização dos elétrons. A banda de energia proibida em polímeros conjugados aumenta ou diminui em função do maior ou menor confinamento dos elétrons $\pi$. Este processo pode ser entendido comparando-o ao comportamento dos níveis de energia em poços quânticos de materiais inorgânicos (por exemplo, GaAs/AlGaAs). O espaçamento entre os níveis de energia em um poço quântico aumenta ou diminui com a diminuição ou aumento da espessura do poço. 


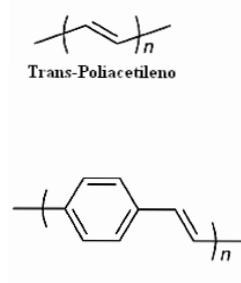

Poli-parafenilenovinileno
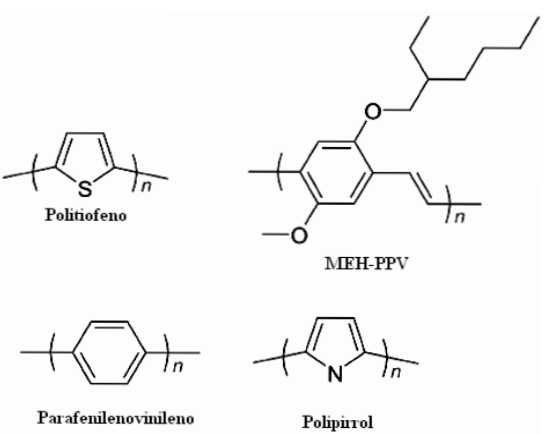

Figura 1. Polímeros conjugados.

O comportamento do "gap" em função da temperatura nos polímeros apresenta um comportamento contrário ao observado em semicondutores inorgânicos. Em binários, como GaAs, InP etc, em ternários como o AlGaAs e em heteroestruturas de GaAs/AlGaAs, o "gap" diminui com o aumento da temperatura, devido aos mecanismos da interação elétron fônon e a dilatação térmica (LOURENÇO et al., 2004). Nos polímeros conjugados, à medida que a temperatura aumenta a cadeia polimérica torna-se mais desordenada, e isso provocando uma maior localização dos elétrons e, conseqüentemente, $\mathrm{E}_{\pi-\pi^{*}}$ aumenta. O comportamento das energias de emissão $\left(\mathrm{E}_{\pi-\pi^{*}}\right)$, da forma de linha dos espectros e das intensidades de emissão em função da temperatura pode fornecer informações importantes sobre propriedades estruturais dos polímeros conjugados, como, por exemplo, o comprimento de conjugação médio das moléculas poliméricas (GETTINGER et al., 1994; OLIVEIRA et al., 2003; PICHLER et al., 1993). Outros fatores que afetam o comportamento do "gap" nos polímeros, como as interações inter-cadeias, ainda não são bem compreendidos, tanto teórica quanto experimentalmente, sendo atualmente objeto de estudos. O estudo do comportamento do "gap" de materiais poliméricos semicondutores, portanto, tem atualmente grande interesse, devido à necessidade de maior compreensão dos fenômenos que ocorrem nos processos de absorção e emissão.

Um dos polímeros semicondutores mais estudados e empregados na fabricação de dispositivos emissores de luz orgânicos OLED ("Organic Light Emithing Diode") é o MEH-PPV (BRAUN; HEEGER, 1991; PARKER, 1994; SCOTT et al., 1996; YANG et al, 2001). Um dos fatores para isto é a sua disponibilidade na forma solúvel, possibilitando, assim, sua preparação em filmes pelas técnicas de "spin-coating" e "dip-coating", entre outras. O MEH-PPV também é um dos polímeros conjugados ativos eletro-opticamente, com grandes possibilidades de utilização na fabricação de lasers orgânicos (KRANZELBINDER; LEISING, 2000).

Neste trabalho, utilizamos um conjunto de três amostras de MEH-PPV preparado por "spincoating" em diferentes rotações (300 rpm, 1000 rpm e $4000 \mathrm{rpm}$ ). Analisamos a influência da velocidade de rotação na posição do pico de PL referente à transição puramente eletrônica (0-0), nos espectros de fotoluminescência obtidos a $300 \mathrm{~K}$ em diferentes posições das amostras e em diferentes intensidades de excitação. Em uma das amostras (1000 rpm), realizamos um estudo da fotoluminescência em função da temperatura no intervalo de $130 \mathrm{~K}$ a $290 \mathrm{~K}$, para estudarmos a variação da posição em energia, da intensidade e da largura de linha a meia altura do pico (0-0) com a temperatura.

Este artigo está organizado da seguinte forma: no item 2 fazemos uma revisão teórica dos conceitos fundamentais necessários para discussão dos resultados, no item 3 apresentamos os pormenores experimentais, e no item 4 mostramos os resultados experimentais e fazemos uma discussão destes resultados. Por fim, no item 5, concluímos nosso trabalho.

\section{Revisão Teórica}

\section{Estrutura Eletrônica e Modelagem}

A origem da banda de energia proibida nos polímeros conjugados pode ser explicada pelo teorema de Peierls (1995). Segundo Peierls, a dimerização da cadeia polimérica leva ao surgimento 
de um "gap" entre os níveis $\pi$ e $\pi^{*}$, dando origem à banda $\pi-\pi^{*}$. No estado fundamental, os elétrons estão emparelhados nos orbitais moleculares de mais baixa energia. O orbital preenchido de mais alta energia na banda $\pi$ (banda de valência) é conhecido como HOMO ("Highest Occupied Molecular Orbital"). Na banda $\pi^{*}$ (banda de condução), o orbital desocupado de mais baixa energia é denominado LUMO ("Lowest Unoccupied Molecular Orbital"). A diferença de energia entre os orbitais LUMO e HOMO nos fornece o "gap" ou $\mathrm{E}_{\pi-\pi^{*}}$. Na Figura 2, ilustramos estes orbitais. A excitação de um elétron de valência para a banda de condução deixa um buraco na banda de valência, gerando um estado excitado do sistema. Os pares elétrons-buraco podem ser não-correlacionados ou correlacionados. Caso sejam correlacionados, existe a formação de éxcitons.

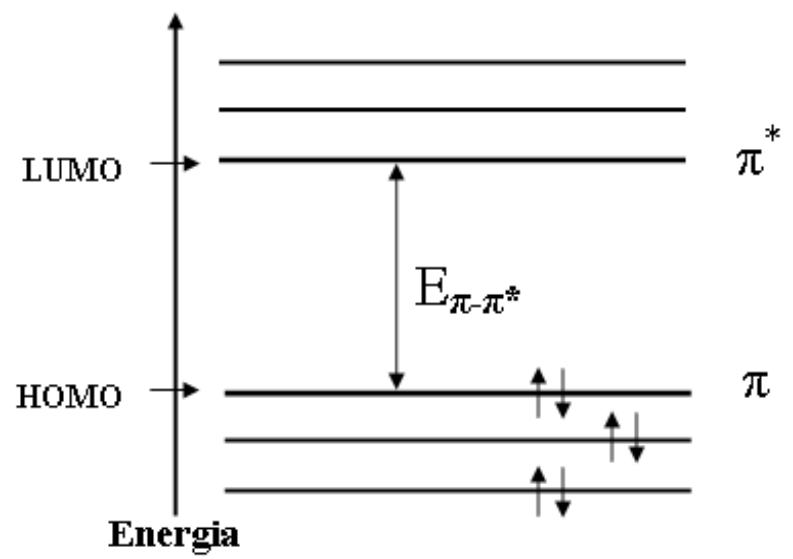

Figura 2. Representação dos orbitais moleculares das bandas $\pi$ e $\pi^{*}$. O orbital preenchido de mais alta energia na banda $\pi$ (banda de valência) é conhecido como HOMO. Na banda $\pi^{*}$ o orbital desocupado de mais baixa energia é denominado LUMO.

Os polímeros conjugados podem ser modelados por uma distribuição estatística de cadeias poliméricas com diferentes comprimentos de conjugação (n). Cada cadeia polimérica possui um comprimento de conjugação diferente. Mostramos na Figura 3, o modelamento de uma única cadeia. Cada segmento conjugado da cadeia é equivalente a um poço quântico unidimensional. Dependendo da extensão destes segmentos, temos poços quânticos de espessuras diferentes, o que influi na diferença de energia entre os estados HOMO e LUMO e, portanto, no "gap" de cada segmento.

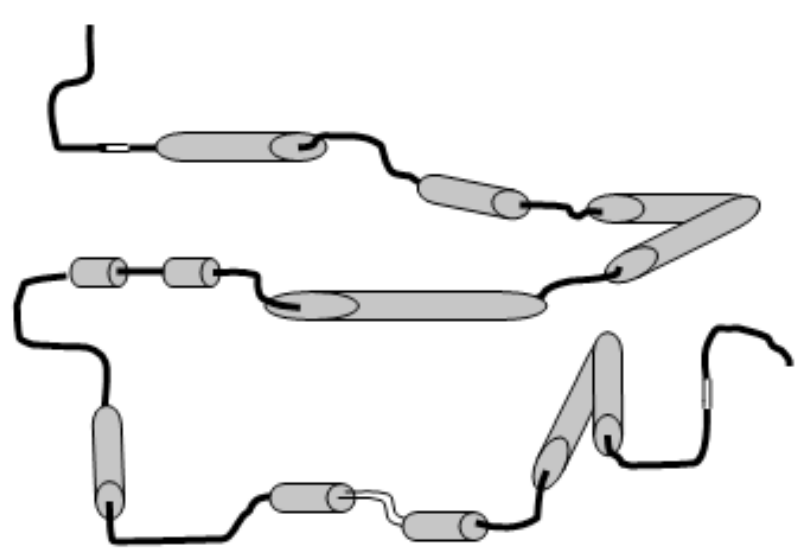

Figura 3. Ilustração do modelo físico proposto para representar uma cadeia polimérica.

Fonte: Oliveira, 2004.

A conjugação pode ser quebrada por defeitos estruturais, torções e presença de segmentos não conjugados. Os tipos mais comuns de defeitos são: o defeito-cis (torção da cadeia), o defeito-sat (saturação da ligação dupla por dois grupos $\mathrm{sp}^{3}$ ) e a oxidação (defeito carbonil) (CHEN; MENG, 2002). A estrutura química do PPV e as ligações dos defeitos são mostrados na Figura 4 (CHEN; MENG, 2002).
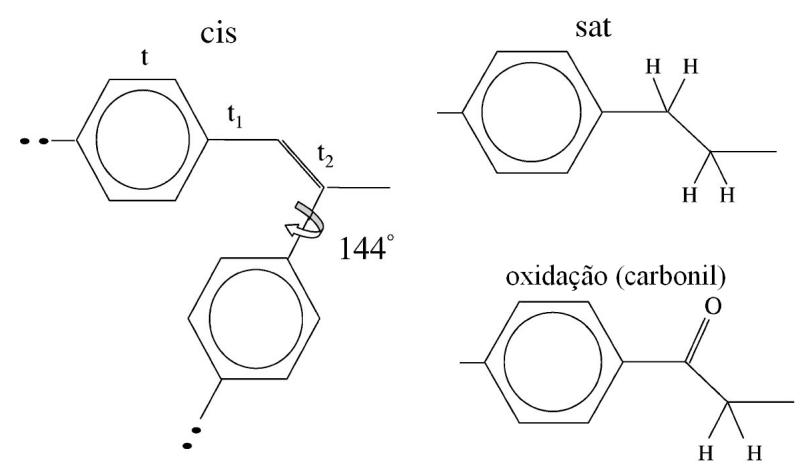

Figura 4. Estrutura química de três tipos de defeitos de conjugação. Existe uma rotação de $144^{\circ}$ para fora do plano no defeito-cis. As ligações C-H mostradas nos defeitos carbonil e -sat são orbitais $\mathrm{sp}^{3}$ saindo do plano. Fonte: Chen e Meng (2002). 
O defeito-cis é uma troca entre o átomo de hidrogênio e o anel fenil conectado a uma ligação dupla vinil. A ligação simples torce e reduz o comprimento de ligação por um fator de $\cos \left(144^{\circ}\right)$. Nos defeitos carbonil e -sat, a conjugação normal $2 \mathrm{p}_{\mathrm{z}}$ é quebrada pela saturação $\mathrm{sp}^{3}$ de um (carbonil) ou dois (-sat) átomos de carbono do esqueleto. Os defeitos-cis são supostamente os que dominam a quebra de conjugação, porque os defeitos de saturação e carbonil podem ser eliminados na síntese do polímero (CHEN; MENG, 2002).

$\mathrm{Na}$ Figura 5, apresentamos uma cadeia polimérica arbitrária com seus vários segmentos conjugados e a estrutura energética de poços quânticos equivalentes. Os portadores de carga ficam confinados nos diferentes poços quânticos, cada qual com sua estrutura característica de níveis de energia discretos. A estrutura eletrônica é, portanto, modulada ao longo da cadeia resultando em espectros de absorção e luminescência bastante largos, comparados com os semicondutores inorgânicos. Isso se deve à incerteza em relação ao comprimento de conjugação das moléculas e ao forte acoplamento dos estados eletrônicos molecular com os estados vibracionais da cadeia.
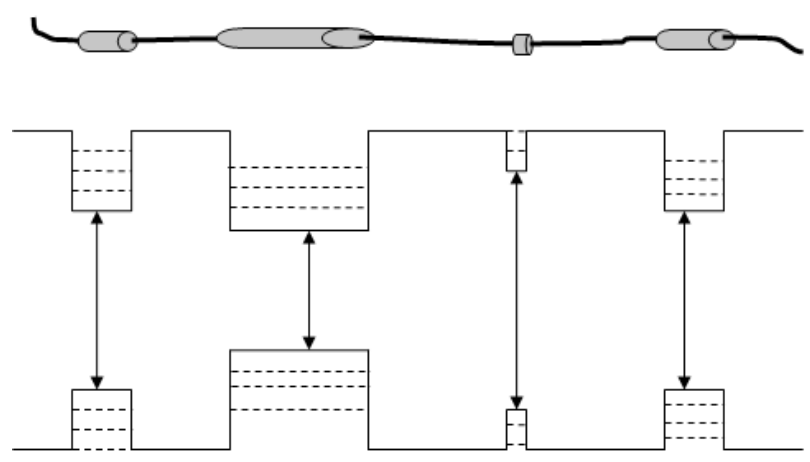

Figura 5. Ilustração do modelo físico utilizado para entendimento qualitativo dos níveis de energia de uma cadeia polimérica conjugada.

Fonte: Oliveira, 2004.

Os estados eletrônicos são fortemente acoplados com as vibrações da cadeia polimérica, fazendo com que os processos de absorção sejam governados pelo princípio de Franck-Condon. Na Figura 6, mostramos a energia potencial, o estado fundamental e o primeiro estado excitado de uma ligação química em um diagrama de configuração, com coordenadas Q (que representam a distância interatômica média dos elementos da molécula). Os níveis vibracionais são quantizados com um intervalo de energia típico de $\sim 0,1 \mathrm{eV}(\sim 1100 \mathrm{~K})$, o que leva, mesmo à temperatura ambiente, que as ligações estejam no nível vibrônico fundamental. Os processos 2-3 e 4-1 (em linhas tracejadas) são associados à interação do elétron com os possíveis modos vibracionais do sistema. As curvas de potencial são deslocadas espacialmente e este deslocamento define a intensidade do acoplamento entre os estados eletrônicos e vibracionais durante a transição óptica, relacionando-se, assim, ao denominado fator de Huang-Rhys (a ser discutido no próximo item). O processo de relaxação 2-3 define o deslocamento em energia entre os espectros de absorção e emissão ("Stokes-shift"). As transições ópticas entre os dois estados moleculares (absorção 1-2 e emissão 3-4) representados por linhas verticais são as transições que caracterizam o princípio de Franck-Condon. As transições verticais porque o movimento dos núcleos é mais lento $\left(\sim 10^{3}\right)$ do que os elétrons.

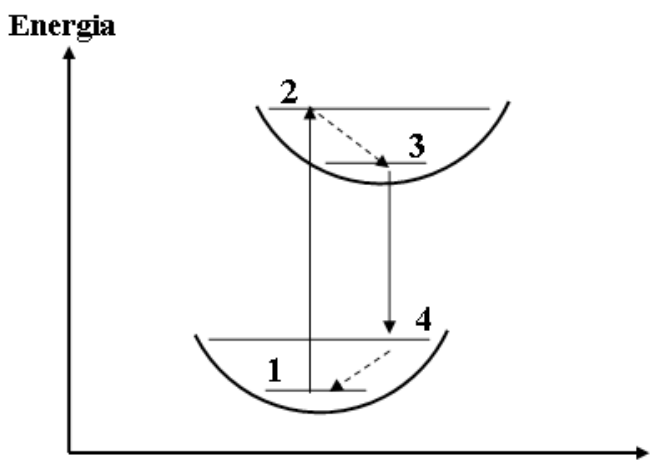

Figura 6. Níveis vibrônicos nos estados eletrônicos, e representação das transições Franck Condon (absorção $1 \rightarrow 2$ e emissão $3 \rightarrow 4$ ). 
Fotofisica dos polímeros conjugados

A fotofísica de materiais orgânicos e não orgânicos é determinada pelas probabilidades dos processos radiativos e não radiativos após a absorção de fótons. Em moléculas orgânicas temos processos intramoleculares e intermoleculares.

\section{Processos Intramoleculares}

O estado fundamental de quase todas moléculas orgânicas é um estado singleto (PHILLIPS, 1985) $\mathrm{S}_{0}$ com elétrons emparelhados com "spins" opostos. A absorção de luz pelos sistemas $\pi$ conjugados resulta na promoção de elétrons da banda $\pi$ para a banda $\pi^{*}$ sem mudança de "spin". O estado excitado criado é também um singleto denominado $\mathrm{S}_{\mathrm{n}}$. Os vários tipos de transições radiativas e não radiativas que podem ocorrer a partir de um estado excitado $\mathrm{S}_{\mathrm{n}}$ em moléculas são representados frequentemente em um diagrama de Jablonski como mostrado na Figura 7. Processos extremamente rápidos $(0.1$ ps) ocorrem na relaxação dos portadores excitados para o estado singleto de mais baixa energia $S_{1}$, por meio da ativação de modos vibracionais (relaxação vibracional RV). Este processo é denominado conversão interna (CI) e leva a um estado de menor energia com mesma multiplicidade de "spin". Após a molécula atingir esse estado, pode ocorrer uma transição para o estado fundamental radiativamente via fluorescência $(F)$ ou não radiativamente via relaxação, por meio de um processo multifônon. Estes processos são competitivos e como o tempo de vida da fluorescência é está entre 1ps e $1 \mathrm{~ns}$, os processos de conversão interna são mais eficientes. Outra possibilidade é a ocorrência de um cruzamento intersistema (CIS) que produz um estado tripleto $T_{n}$. Nesse caso a multiplicidade do "spin" é invertida através da interação "spin órbita" (GULLET, 1987) Processos de conversão interna levam o estado tripleto excitado a relaxar para um estado tripleto de mais baixa energia, $\mathrm{T}_{1}$, e, a partir daí, a relaxação/transição para o estado fundamental pode ocorrer através de processos radiativos, fosforescência (FOS) ou fluorescência, após um outro processo CIS ou outros processos não radiativos como relaxação vibracional. A eficiência do processo de fosforescência é baixa devido ao seu grande tempo de vida $(\tau)$, da ordem de $\sim 1 \mu$ s. A análise comparativa destes processos competitivos nos fornece:

$$
\frac{1}{\tau_{C I}} \approx \frac{1}{\tau_{C I S}}>>\frac{1}{\tau_{F}}>>\frac{1}{\tau_{F O S}}
$$

onde $\tau$ é o tempo de vida da transição, os subíndices CI, CIS, F e FOS são os processos de conversão interna, conversão intersistema, fluorescência e fosforescência, respectivamente. Os elétrons nos estados $\mathrm{S}_{1}$ e $\mathrm{T}_{1}$ podem ligar-se a buracos e formar os éxcitons singletos ou tripletos e então recombinarem.

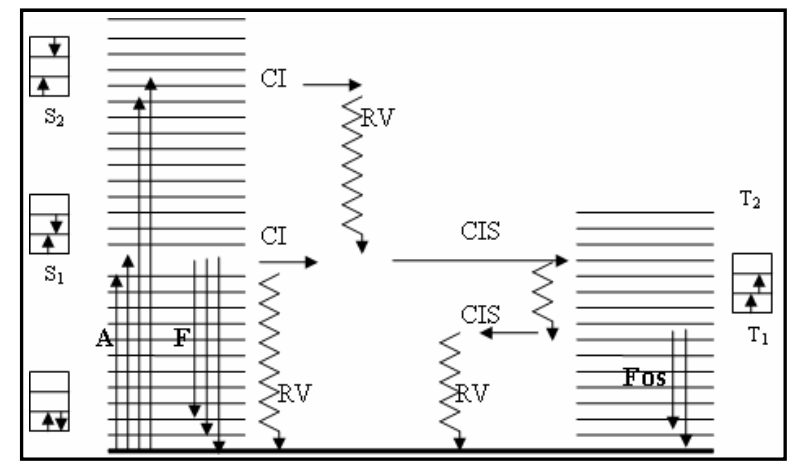

Figura 7. Diagrama de Jablonski representando os processos de recombinação intramoleculares após a fotoexcitação. As setas onduladas indicam geração de fônons e as setas retilíneas envolvem fótons.

Fonte: Oliveira, 2004.

\section{Processos Intermoleculares}

Existem diferentes processos intermoleculares não radiativos que competem com os processos radiativos e não-radiativos intramoleculares. Vários mecanismos são propostos para explicar a transferência de energia de forma não-radiativa de segmentos de menor conjugação para segmentos de maior conjugação (difusão espectral) em polímeros conjugados (GULLET, 1987; GREENHAM; 
FRIEND, 1955; FRIEND; BRADLEY; TOWNSEND, 1987; MARLETTA et al., 2001). Entre estes mecanismos podemos mencionar a migração por "hopping" e o mecanismo Föster.

A migração por "hopping" consiste no tunelamento dos portadores de carga entre moléculas de diferentes comprimentos de conjugação, o que exige superposição das funções de onda do estado inicial e final. $\mathrm{O}$ mecanismo Föster não exige a superposição de funções de ondas e é o mais aceito atualmente para explicar a difusão espectral.

A migração ou transferência de Föster ocorre através da doação de energia de uma molécula excitada (doadora $-\mathrm{D}^{*}$ ) para outra molécula no estado fundamental (aceitadora - $\mathrm{A}^{*}$ ). A interação entre as moléculas doadoras e aceitadoras pode ser representada pela equação:

$$
D^{*}+A \rightarrow D+A^{*}
$$

onde $\mathrm{D}$ e $\mathrm{A}$ representam as moléculas doadora e aceitadora ano estado fundamental e $D^{*}$ e $A^{*}$ representam as moléculas doadora e aceitadora no estado excitado. Uma representação da transferência de energia pelo mecanismo Föster pode ser vista na Figura 8.

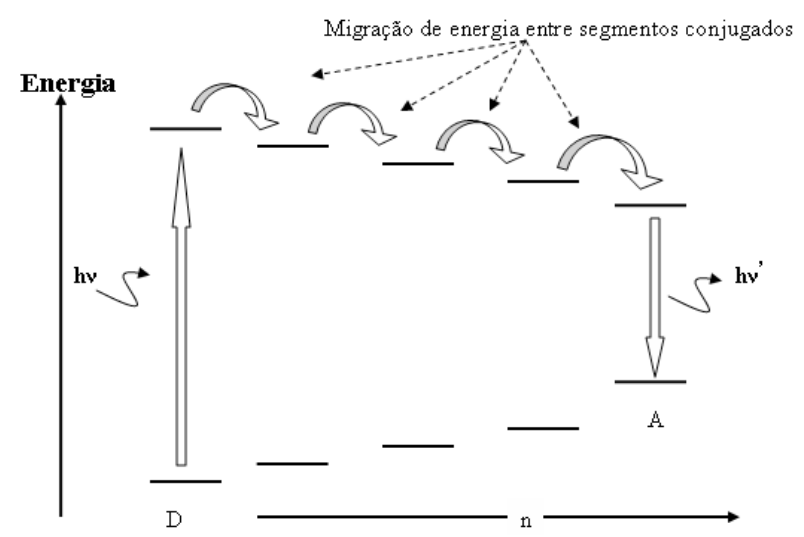

Figura 8. Representação da transferência de energia pelo mecanismo de Föster entre moléculas conjugadas com diferentes graus de conjugação.

Fonte: Oliveira, 2004.
Após a excitação óptica, a molécula excitada pode transferir energia para uma molécula vizinha com maior grau de conjugação. Recebida essa energia, a molécula vizinha passa para o estado excitado e pode transferir energia para uma outra que eventualmente apresenta um grau ainda maior de conjugação. Este processo ocorre até que a transferência de energia para uma determinada molécula, na qual a probabilidade de transição radiativa seja maior que a probabilidade de transição não radiativa via mecanismo de Föster, se dê, havendo então a emissão de luz.

\section{Processos Ópticos em Função da Temperatura}

O tratamento teórico dos processos fotofísicos que surgem nos polímeros devido a excitações ópticas pode ser desenvolvido utilizando a aproximação de Born-Oppenheimer.

Consideremos uma transição radiativa entre um estado eletrônico fundamental a e um estado excitado b. Usando o modelo de coordenadas configuracionais, em uma aproximação harmônica, mostramos na Figura 9 as funções de onda na aproximação de Born-Oppenheimer que descrevem os estados fundamental e excitado, respectivamente $\psi_{a}(r, R) \chi_{a}(n)$ e $\psi_{b}(r, R) \chi_{b}(m)$. Para simplificar, considera-se que as freqüências vibracionais dos estados fundamental e excitado são iguais permitindo-se, entretanto que o valor médio das coordenadas de configuração, Q, sejam diferentes. A diferença no valor médio de $\mathrm{Q}$ surge devido às diferenças no acoplamento elétron - fônon entre os dois estados a e b. A diferença entre as energias dos estados a e b pode ser dada por:

$$
E^{b}(Q)-E^{a}(Q)=E_{a b}-A \hbar \omega\left(\frac{M \omega}{\hbar}\right)^{\frac{1}{2}}\left(Q-Q_{0}^{(a)}\right)
$$

onde $E^{a}(Q)=0$ (energia do estado fundamental), $E^{b}(Q)$ é a energia do estado excitado, $E_{a b}$ é a energia de absorção e A é dado por: 


$$
A=\left(\frac{M \omega}{\hbar}\right)^{\frac{1}{2}}\left(Q_{0}^{(b)}-Q_{0}^{(a)}\right)
$$

e caracteriza a diferença no acoplamento elétron fônon entre os estado eletrônicos a e b. $Q_{0}^{(b)}$ e $Q_{0}^{(a)}$ são respectivamente as coordenadas dos mínimos das superfícies de energia de potencial que descrevem os estados fundamental e excitado. Pode-se representar A em termos de uma outra constante adimensional, a constante de Huang-Rhys, S, definida por:

$$
S=\frac{A^{2}}{2}=\frac{1}{2} \frac{M \omega^{2}}{\hbar \omega}\left(Q_{0}^{(b)}-Q_{0}^{(a)}\right)^{2}=\frac{E_{d i s}}{\hbar \omega}
$$

onde $E_{d i s}$ é a energia dissipada após a absorção. O fator $\mathrm{S}$ mede, portanto, o deslocamento relativo entre os mínimos das curvas de potencial do estado fundamental e do excitado. Para cadeias com pequeno grau de conjugação, o espaçamento entre a banda fundamental e excitada é grande, e isso implica em um fator de Huang-Rhys alto. Para cadeias com grande grau de conjugação o espaçamento é menor e o fator de Huang-Rhys é baixo.

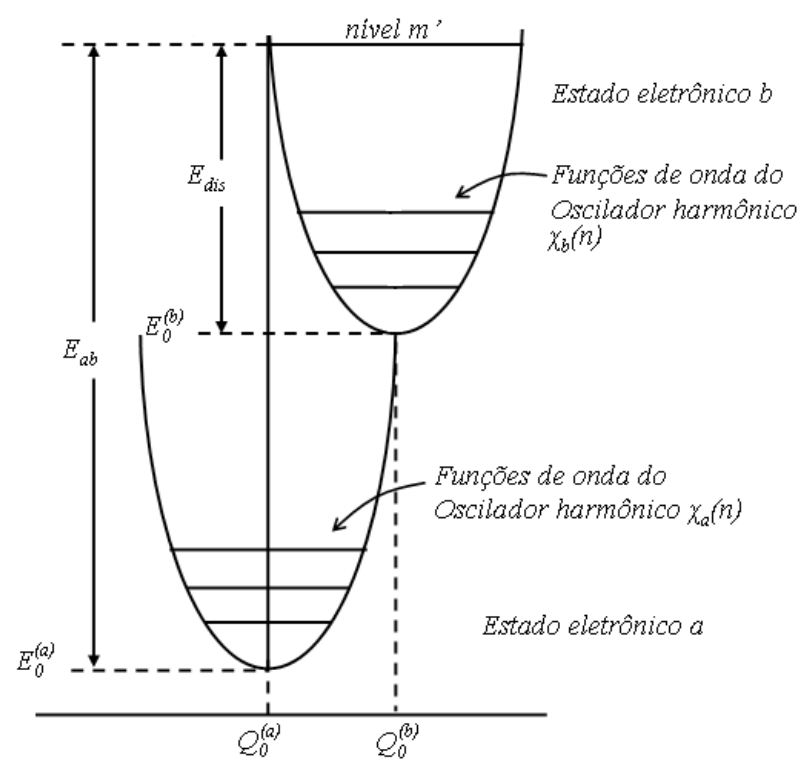

Figura 9. Diagrama configuracional de coordenadas em uma aproximação harmônica para os estados eletrônicos a e b (OLIVEIRA, 2004).

\section{Análise de Frank-Condon}

A probabilidade de transição entre um estado eletrônico-vibracional inicial $\left|\chi_{a}(n)\right\rangle$ para um estado eletrônico-vibracional final $\left|\chi_{b}(m)\right\rangle$ é proporcional ao quadrado do elemento de matriz entre estes dois estados.

Considerando a existência de apenas um modo vibracional de energia $\hbar \omega$ com o qual o espectro óptico pode acoplar, a forma de linha para a banda de absorção para uma transição $\left|\chi_{a}(n)\right\rangle \rightarrow\left|\chi_{b}(m)\right\rangle$ pode ser expressa pela regra de ouro de Fermi (LIN, 1973)

$$
I=I_{0} \sum_{n} \sum_{m} A v_{n}\left|\left\langle\chi_{b}(m) \| \chi_{a}(n)\right\rangle\right|^{2} \delta\left(E_{b, m}-E_{a, n}-E\right)
$$

onde $\mathrm{A} v_{\mathrm{n}}$ (fator de Boltzman) indica a ocupação térmica sobre o $n$-ésimo estado vibracional, $E_{a, n}$ e $E_{b, m}$ são as energias do $n$-ésimo e $m$-ésimo estados vibracionais nos estados eletrônicos a e b, respectivamente. Esta banda consistirá de funções delta centralizadas em energias múltiplas de $\hbar \omega$. Se o alargamento de linha é significativo, a função delta é trocada por uma função distribuição que leve em conta a dispersão energética em torno de $E_{a, n} \mathrm{e}$ $E_{b, m}(\mathrm{LIN}, 1973)$.

Em processos que envolvem a absorção por moléculas a integral de superposição das funções de onda vibracionais é obtida considerando o princípio Franck-Condon (LIN, 1971; KEIL, 1965) e pode ser expressa na forma,

$$
\left\langle\chi_{b}(m) \| \chi_{a}(n)\right\rangle=\mathrm{e}^{-\frac{A^{2}}{4}}\left(\frac{n !}{m !}\right)^{\frac{1}{2}}\left(-\frac{A}{\sqrt{2}}\right)^{m-n} L_{n}^{m-n}\left(-\frac{A^{2}}{2}\right)
$$

onde $L_{n}^{m-n}$ são os polinômios de Laguerre (no caso específico de $n=0$ temos que $\left.L_{0}^{m}(x)=1\right)$ e

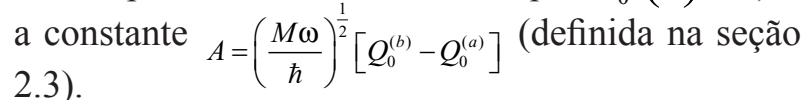


Para $\mathrm{T}=0 \mathrm{~K}$ somente o estado vibracional $n=$ 0 está ocupado, e o fator Franck-Condon assume a forma:

$$
F_{m}(0)=\left|\left\langle\chi_{b}(m)|| \chi_{a}(n)\right\rangle\right|^{2}=\mathrm{e}^{-\frac{A^{2}}{4}}\left(\frac{A^{2}}{2}\right)^{m} \frac{1}{m !}
$$

que em termos do parâmetro S de Huang-Rhys, definido na Equação 2.5, pode ser reescrito como,

$$
F_{m}(0)=\mathrm{e}^{-S}(S)^{m} \frac{1}{m !} .
$$

Assim, em $\mathrm{T}=0 \mathrm{~K}$ a função que molda a banda de absorção é

$$
I_{a b}(E)=I_{0} \sum_{m}\left(\frac{\mathrm{e}^{-S} S^{m}}{m !}\right) \delta\left(E_{b, m}-E_{a, 0}-E\right),
$$

sendo $E_{b, m}=E_{b, 0}+m \hbar \omega$ e $E_{b 0}-E_{a 0}=E_{0}$, temos

$$
I_{a b}(E)=I_{0} \sum_{m}\left(\frac{\mathrm{e}^{-S} S^{m}}{m !}\right) \delta\left(E_{0}+m \hbar \omega-E\right) .
$$

Para a emissão, após a excitação do sistema para algum estado vibracional $m$ no estado eletrônico b, ocorre decaimento não-radiativo via um processo multifônon para o estado $\chi_{b}(0)$. Após este decaimento não-radiativo, segue-se a emissão de radiação eletromagnética, levando o sistema para o estado $\chi_{a}(n)$. Semelhantemente à absorção, a forma da banda de emissão a temperatura $\mathrm{T}=0$ K é

$$
I_{b a}(E)=I_{0} \sum_{n}\left(\frac{\mathrm{e}^{-S} S^{n}}{n !}\right) \delta\left(E_{0}-n \hbar \omega-E\right) .
$$

A linha que corresponde às transições puramente eletrônicas tem intensidade de:

$$
I_{00}=I_{0} \mathrm{e}^{-S},
$$

onde o subscrito 00 indica transição óptica sem acoplamento com modos vibracionais. Para $m=1$ a intensidade é dada por,

$$
I_{01}=I_{0} \mathrm{e}^{-S} S,
$$

onde o subscrito 01 indica transição óptica envolvendo o acoplamento com um único modo vibracional. Portanto, dividindo-se a Equação 2.14 pela equação 2.13 temos:

$$
S=\frac{I_{01}}{I_{00}}
$$

que nos permite obter experimentalmente o fator de Huang-Rhys. Para a interação com mais de um modo vibracional pode ser utilizada a progressão de Franck-Condon da linha de 00 (HAGLER et al., 1991; MOSES et al., 1982) que é dada por

$$
I_{b a}(E)=I_{0} \sum_{n_{i}}\left(\frac{\prod_{i}^{m} \mathrm{e}^{-S_{i}} S_{i}^{n_{i}}}{n_{i} !}\right) \delta\left(E_{0}-\sum_{i} n_{i} \hbar \omega_{i}-E\right)
$$

Assim, para cada modo vibracional, haverá um fator Huang-Rhys diferente $\mathrm{S}_{1}, \mathrm{~S}_{2}, \mathrm{~S}_{3}, \ldots$ e cada um pode ser obtido através da equação 2.15 utilizando a intensidade $I_{0 i}$ respectiva à transição assistida pelo modo vibracional de freqüência $\omega_{i}$.

Propriedades Ópticas de Polímeros Conjugados e sua Dependência com a Temperatura

Os espectros de absorção e emissão dos polímeros conjugados, diferentemente de materiais inorgânicos, não apresentam uma forma de linha 
estreita e bem resolvida. Isso se deve à incerteza em relação ao comprimento de conjugação das moléculas e ao forte acoplamento dos estados eletrônicos moleculares com os estados vibracionais da cadeia.

As transições ópticas em polímeros conjugados podem ser basicamente descritas por cinco parâmetros:

1 - a posição em energia do pico da transição puramente eletrônica (0-0) $E_{0}$;

2 - a intensidade da linha $I_{00}$ da transição puramente eletrônica;

3 - a largura de linha a meia altura $\Gamma$ da transição puramente eletrônica;

4 - o fator Huang-Rhys para cada modo vibracional $S_{i}$

5 - as energias dos modos vibracionais $\hbar \omega_{i}$ que acoplam com as transições eletrônicas.

Conhecendo esses parâmetros, é possível reproduzir inteiramente o comportamento dos espectros de absorção e de emissão. A largura a meia altura (Г) FWHM ("Full Width Half Maximum”) fornece a dispersão do comprimento de conjugação das cadeias absorvedoras e emissoras. A intensidade da linha (0-0), o fator Huang-Rhys e a energia dos modos vibracionais fornecem as intensidades de todos os picos do espectro bem como a sua distância em relação à energia do pico da transição puramente eletrônica(0-0). Neste trabalho, estamos interessados na dependência dos parâmetros enumerados acima com a temperatura.

A cadeia polimérica é composta por segmentos conjugados com diferentes graus de conjugação n. Desse modo, os estados eletrônicos são profundamente afetados pela extensão espacial da função de onda. Empiricamente, a dependência da energia de transição da linha (0-0) com o grau de conjugação n (YU et al., 1996; WOO et al., 1993; HENLEY; KOHLER, 1977) pode ser escrita como $E=E_{0}+\Delta / n$, onde $\mathrm{E}_{0}$ é a menor energia de "gap" no caso de absorção ou de emissão a temperatura de $0 \mathrm{~K}$, e $\Delta$ é um parâmetro que depende do material polimérico. No entanto, torções moleculares provocadas pela desordem térmica reduzem o tamanho efetivo de conjugação devido ao desvio da planaridade da cadeia. A dependência do grau de conjugação $\mathbf{n}$ com a temperatura pode ser descrita em termos da energia conformacional responsável pelas torções moleculares através da expressão $n=n_{0} \exp \left(\varepsilon / k_{B} T\right)$, onde $\mathbf{n}_{0}$ é o grau de conjugação efetivo a altas temperaturas. Deste modo, a dependência com a temperatura da energia do pico puramente eletrônico é dada por:

$$
E=E_{0}+\frac{\Delta E}{\exp \left(\varepsilon / k_{B} T\right)}
$$

onde $\mathrm{E}_{0}$ é o "gap" de energia em $\mathrm{T}=0 \mathrm{~K}, \Delta \mathrm{E}=\Delta / \mathrm{n}_{0}$ indica a variação máxima de energia da linha $(0-0)$ no processo de emissão e $\varepsilon$ é a energia necessária para efetuar as mudanças conformacionais (rotações entre monômeros, dobramentos, estiramentos,...) nas moléculas poliméricas.

A intensidade do pico puramente eletrônico em função da temperatura é dada por:

$$
I_{00}=\frac{I_{00}(0)}{1+B \exp \left(-E_{a t} / k_{B} T\right)}
$$

onde $\mathrm{I}_{00}(0)$ é a intensidade em $\mathrm{T}=0 \mathrm{~K}$, B é um parâmetro de ajuste, $\mathrm{E}_{\mathrm{at}}$ é a energia de ativação dos processos não radiativos e $k_{B}$ é a constante de Boltzmann.

A contribuição da parte homogênea do alargamento espectral é de natureza dinâmica devido as flutuações térmicas da cadeia (torções) e acontece em cada sítio da amostra. A variação da parte homogênea da largura de linha a meia altura (FWHM) da transição puramente eletrônica (0-0) com a temperatura é dada pela equação de acordo com (NABETANI et al., 1995):

$$
\Gamma(T)=\Gamma_{0} \operatorname{coth}\left(\frac{\hbar \omega}{2 k_{B} T}\right)
$$


onde $\Gamma_{0}$ é a largura em $\mathrm{T}=0 \mathrm{~K}$, ou seja a contribuição da parte não homogênea do alargamento, $\hbar \omega$ é a energia de um modo vibracional, $k_{B}$ é a constante de Boltzmann.

A variação do acoplamento elétron-fônon com a temperatura é muito importante, pois o perfil espectral é alterado por esta dependência. As amplitudes relativas da estrutura vibracional e linha puramente eletrônica no espectro de emissão são medidas desse acoplamento, que é definido pelo fator Huang-Rhys, S. Este parâmetro $\mathbf{S}$ também quantifica o número médio de fônons envolvidos no processo de relaxação. Estudos experimentais indicam que o parâmetro $\mathbf{S}$ está relacionado com a estrutura do sistema molecular, sendo maior em sistemas que apresentam estados eletrônicos localizados (segmentos conjugados de menor tamanho).

Uma relação entre o fator Huang-Rhys $\mathbf{S}$ e o grau de conjugação n é dado por: (HAGLER et al., 1991; PEETERS et al., 2000)

$$
S_{i}=a_{i}+\frac{b_{i}}{n_{0} \exp \left(\varepsilon / k_{B} T\right)+1}
$$

onde $i=1,2,3, \ldots, a_{i}$ é o fator Huang-Rhys para moléculas com alto grau ou tamanho de conjugação efetivo grande, $\mathbf{n}_{0}$ é o grau de conjugação e $b_{i}$ é um parâmetro a ser determinado.

Zeng e Ding (2004) utiliza uma outra relação entre o fator Huang-Rhys $\mathbf{S}$ e o grau de conjugação n é dada por:

$$
S=a \exp \left(-n^{2} / b\right)
$$

onde $a, b$ são empiricamente escolhidos com valores de 3.2 e 38 (YU et al., 1996) respectivamente.
As energias de fônons (modos vibracionais) podem ser obtidas por medidas de Raman (WANTZ et al., 2005) e infravermelho. Na Figura 10 apresentamos o espectro de Raman do, polímero conjugado derivado do PPV, BDMO-PPV que segundo Wantz et al. (2005) é idêntico ao espectro de MEH-PPV obtido nas mesmas condições experimentais.

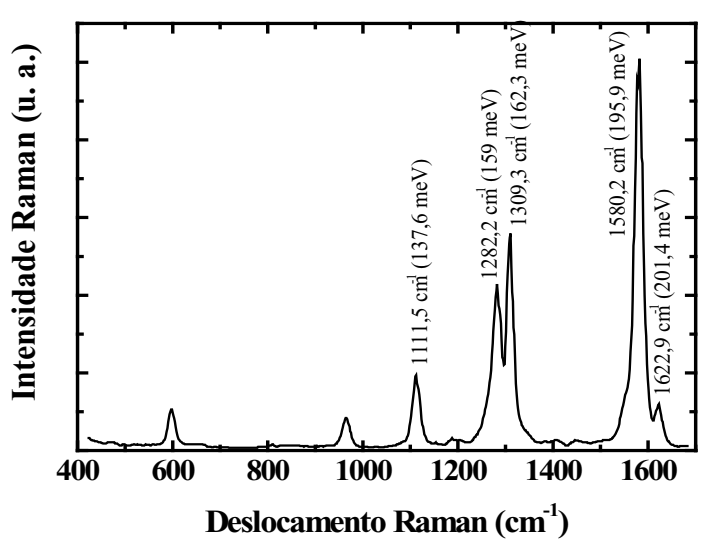

Figura 10. Espectro de Raman do BDMO-PPV obtido na temperatura ambiente $(300 \mathrm{~K})$ foi redigitalizado do trabalho de Wantz et al. (2005)

Na Tabela 1, apresentamos os principais modos vibracionais obtidos por Raman a $\mathrm{T}=300 \mathrm{~K}$ para o MEH-PPV, conforme pode ser visto no espectro da Figura 10. 
Tabela 1. Principais modos vibracionais obtidos por Raman a $\mathrm{T}=300 \mathrm{~K}$ para o MEH-PPV.

\begin{tabular}{lll}
\hline \multicolumn{1}{c}{ Estrutura } & $v\left(\mathrm{~cm}^{-1}\right)$ & $v(\mathrm{eV})$ \\
\hline Estiramento C=C do grupo vinil & 1622.9 & 201.4 \\
Estiramento C-C do anel fenílico & 1580.2 & 195.9 \\
Estiramento C=C + dobramento C-H do grupo vinil & 1309.3 & 162.3 \\
Estiramento C-C + dobramento C-H do anel fenílico & 1111.5 & 137.8 \\
\hline
\end{tabular}

\section{Detalhes Experimentais}

Os filmes poliméricos de MEH-PPV estudados neste trabalho foram preparados pela técnica de "spin-coating”, em uma concentração de $10 \mathrm{mg} / \mathrm{ml}$ de xileno sobre substratos de vidros com uma taxa de rotação diferente para cada amostra, amostra A4000 $=4000 \mathrm{rpm}$, amostra A1000 = $1000 \mathrm{rpm}$ e amostra $\mathrm{A} 300=300 \mathrm{rpm}$. Os substratos de vidro foram limpos em ultra-som com acetona por 20 minutos e depois com água deionizada por 10 minutos e secos com $\mathrm{N}_{2}$. A análise da emissão em função da temperatura foi efetuada com o emprego da técnica de fotoluminescência com excitação utilizando a linha de $458 \mathrm{~nm}$ de um laser de argônio $\left(\mathrm{Ar}^{+}\right)$. O sinal é analisado por um monocromador Jarrel-Ash $0.5 \mathrm{~m}$ e detectado por uma fotomultiplicadora de GaAs usando técnicas padrão de Lock-in.

As amostras foram acomodadas sob vácuo dentro de um criostato e presas a um porta-amostras de acordo com a Figura 11a. O porta-amostras está em contato com um dedo frio refrigerado por um circuito fechado de He. Considerando a péssima condutividade térmica do vidro, é de se esperar que não haja um bom contato térmico entre o dedo frio e o filme polimérico na configuração da Figura 11a. Sendo assim, o sensor de temperatura foi posto sobre o filme, com a intenção de se obter uma leitura mais adequada da temperatura do filme. A mais baixa temperatura do filme verificada com esta configuração foi de $130 \mathrm{~K}$, mesmo com o dedo frio sendo refrigerado até $10 \mathrm{~K}$ (o limite inferior de temperatura obtido com o sistema de circuito fechado de He empregado nestas medidas).

Para testar a confiabilidade da leitura da temperatura do filme feita na configuração da Figura 11a, utilizamos outras configurações experimentais, como mostrados na Figura 11b e Figura 11c. Na configuração da Figura 11b, o filme foi colocado em contato térmico com o porta-amostras, assim como o sensor de temperatura. O filme foi excitado pelo laser através de um orifício feito no porta-amostras, e a emissão foi coletada por meio do substrato de vidro. Na configuração da Figura 11c, o filme foi depositado sobre um substrato de cobre (seguindo as mesmas condições de deposição que o anterior), e o substrato de $\mathrm{Cu}$ foi posto em contato térmico com o porta-amostras. Outros estudos mostram que filmes de MEH-PPV depositados sob estas condições não são influenciados pela natureza metálica do substrato (SILVA et al., 2008). Nas configurações (b) e (c), a temperatura do dedo frio foi mantida em $130 \mathrm{~K}$, com o intuito de comparar com a situação da Figura 11a.

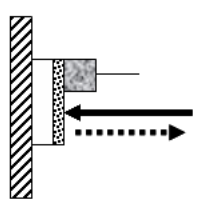

(a)

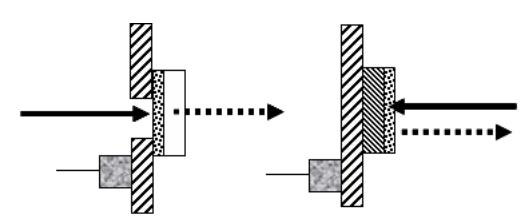

(b) (c)

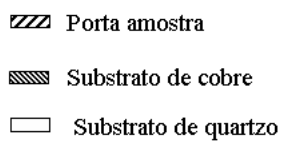

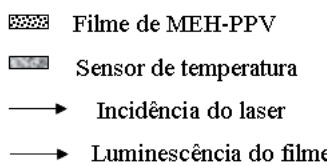

Figura 11. Configurações experimentais das medidas de fotoluminescência para determinação da melhor maneira de se controlar a temperatura durante a realização de medidas quando o substrato é um isolante térmico, como o vidro. 
$\mathrm{Na}$ Figura 12 apresentamos os espectros de fotoluminescência obtidos nas diferentes configurações experimentais mostradas na Figura 11.

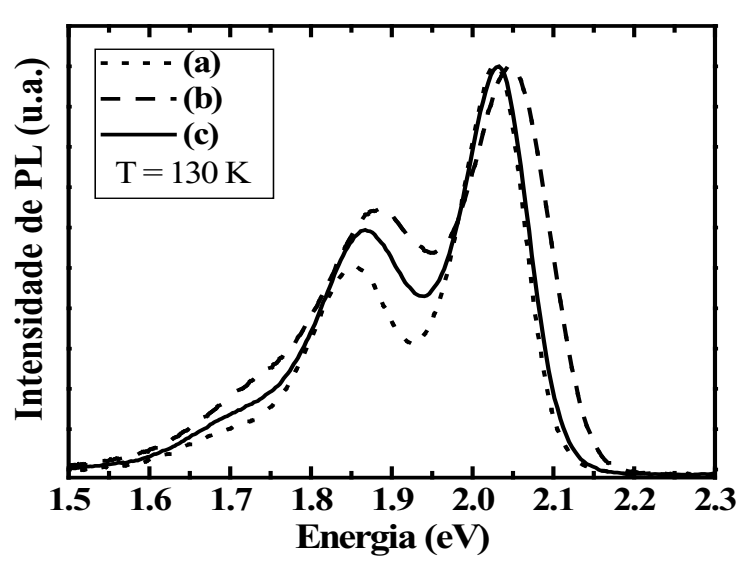

Figura 12. Espectros de fotoluminescência medidos a $T$ $=130 \mathrm{~K}$ para as configurações mostradas na Figura 11. A linha pontilhada representa a medida realizada no MEHPPV sobre vidro com o sensor de temperatura sobre o filme. A linha tracejada representa a medida realizada no MEH-PPV sobre vidro com o sensor de temperatura sobre o porta-amostras e o filme em contato com o portaamostras. A linha contínua representa a medida realizada no MEH-PPV sobre cobre com o sensor de temperatura sobre o porta-amostras.

Podemos avaliar a temperatura do filme pela posição em energia do pico puramente eletrônico. Sendo assim, os espectros sugerem que:

i) a temperatura do filme nas configurações (a) e (c) é equivalente. Dessa forma, utilizaremos a configuração (a) para realizarmos a variação de temperatura na amostra A1000.

ii) o filme na configuração (b) está em uma temperatura superior à das outras configurações (o pico (0-0) tem energia maior), mostrando que esta configuração não representa a verdadeira temperatura do filme.

iii) a temperatura mínima confiável em que podemos realizar medidas de fotoluminescência no filme de MEH-PPV depositado sobre vidro utilizando o sistema de resfriamento existente em nosso laboratório é de aproximadamente $130 \mathrm{~K}$. Nossas medidas de PL foram realizadas no intervalo de 130 a $290 \mathrm{~K}$.

\section{Resultados experimentais e discussão}

$\mathrm{Na}$ Figura 13 apresentamos os espectros de fotoluminescência das amostras A4000, A1000 e A300 a temperatura ambiente $(300 \mathrm{~K})$ e intensidade de excitação de $1.7 \mathrm{~mW} / \mathrm{cm}^{2}$.

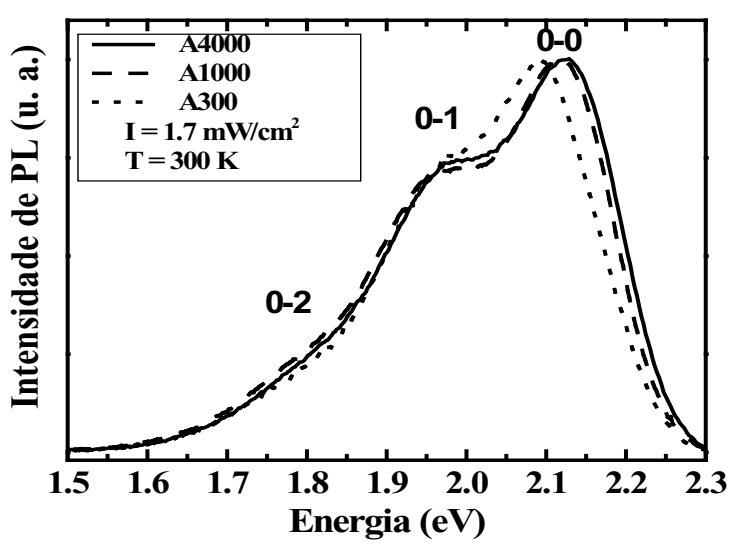

Figura 13. Espectros de PL obtidos a temperatura ambiente $(\mathrm{T}=300 \mathrm{~K})$ e intensidade de excitação de 1.7 $\mathrm{mW} / \mathrm{cm}^{2}$. A linha contínua representa o espectro de PL da amostra A4000, a linha tracejada representa o espectro de PL da amostra A1000 e a linha pontilhada representa o espectro de PL da amostra A300.

Os espectros de fotoluminescência apresentam 2 picos na região de alta energia e um ombro na região de baixa energia. O pico principal (0-0) em $2.12 \mathrm{eV}$ está associado a uma transição puramente eletrônica, o segundo pico (0-1) em 1.98 eV está associado a primeira banda vibrônica, e o ombro (0-2) em aproximadamente $1.78 \mathrm{eV}$ está associado a segunda banda vibrônica. Note-se que, para a amostra A300 (linha pontilhada), o pico (0-0) está um pouco deslocado para menor energia, de aproximadamente $30 \mathrm{meV}$, em relação ao pico equivalente da amostra A4000. Este comportamento está possivelmente relacionado à diminuição da espessura do filme e ao aumento de sua homogeneidade com o aumento da rotação. 
A homogeneidade do filme ao longo da amostra foi verificada através de medidas de fotoluminescência em três pontos diferentes de cada amostra a temperatura ambiente $(300 \mathrm{~K})$ e sob mesma intensidade de excitação $\left(1.7 \mathrm{~mW} / \mathrm{cm}^{2}\right)$. Os resultados destas medidas para as três amostras são apresentados na Figura 14. Consideramos o centro da amostra a posição $0 \mathrm{~mm}$ com valores $>0$ acima e valores $<0$ abaixo do centro. Apresentamos na Figura 14a as medidas realizadas no centro, a 2 $\mathrm{mm}$ (linha pontilhada) e a $6 \mathrm{~mm}$ (linha tracejada) do centro da amostra A4000. Na Figura 14b, apresentamos as medidas realizadas no centro, a 6 $\mathrm{mm}$ (linha pontilhada) e a $-6 \mathrm{~mm}$ (linha tracejada) do centro da amostra A1000. Apresentamos na Figura $14 \mathrm{c}$ as medidas realizadas no centro e a -6 mm (linha tracejada) do centro da amostra A300.

A amostra que apresenta maior homogeneidade é a A4000, de maior rotação. Este é um resultado esperado, uma vez que o aumento da velocidade de rotação na preparação dos filmes faz aumentar sua homogeneidade.

Na Figura 15 mostramos o comportamento dos espectros de fotoluminescência dos três filmes obtidos na posição central da amostra com diferentes intensidade de excitação a temperatura ambiente (300 K).

A variação da intensidade de excitação não influencia a posição do pico principal (0-0), pelo menos a $300 \mathrm{~K}$. Nota se que a variação da intensidade de excitação altera (muito pouco) a intensidade da primeira banda vibrônica (0-1), principalmente na amostra A300 preparada com menor rotação.
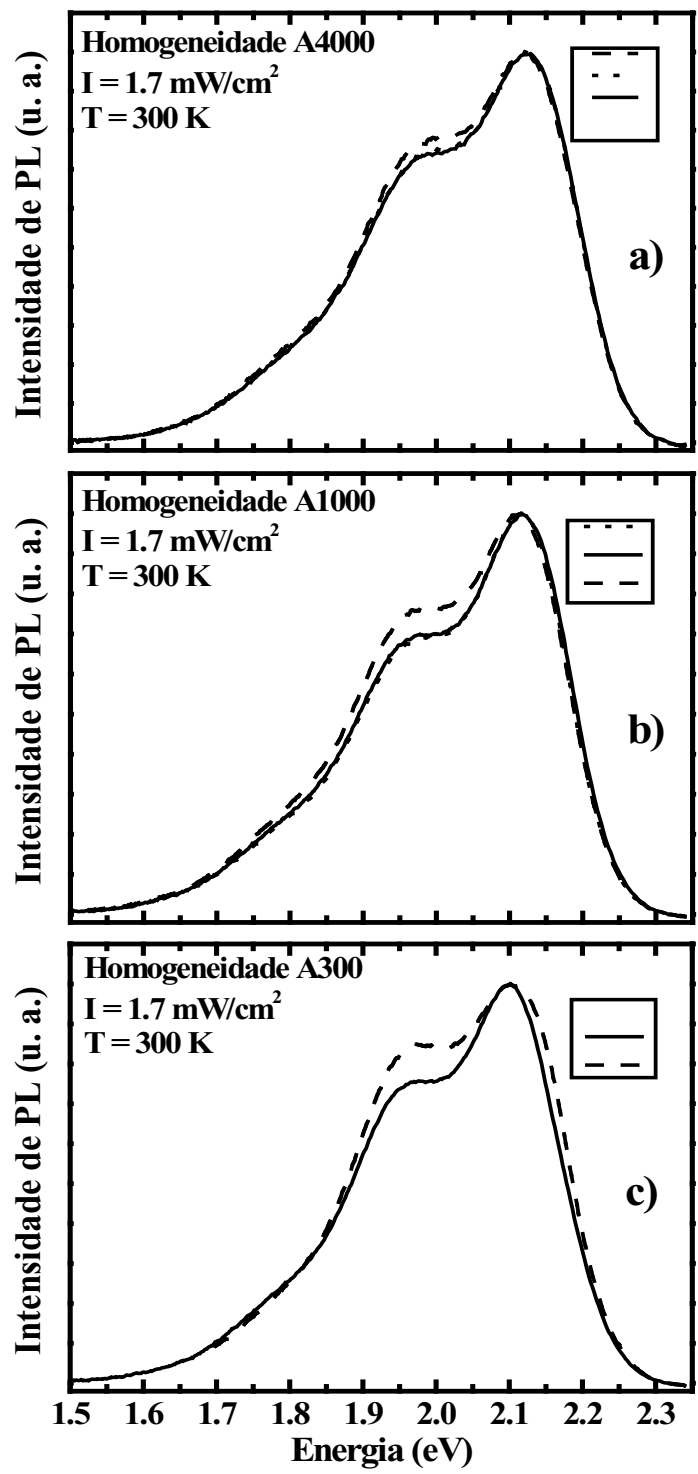

Figura 14. Homogeneidade dos filmes a temperatura ambiente $(\mathrm{T}=300 \mathrm{~K})$ e intensidade de excitação $(1.7$ $\mathrm{mW} / \mathrm{cm}^{2}$ ). a) Medidas realizadas na amostra A4000. b) Medidas realizadas na amostra A1000. c) Medidas realizadas na amostra A300. A posição das linhas dentro dos quadros a direita dos espectros representa a posição na amostra onde foram feitas as medidas de fotoluminescência. 

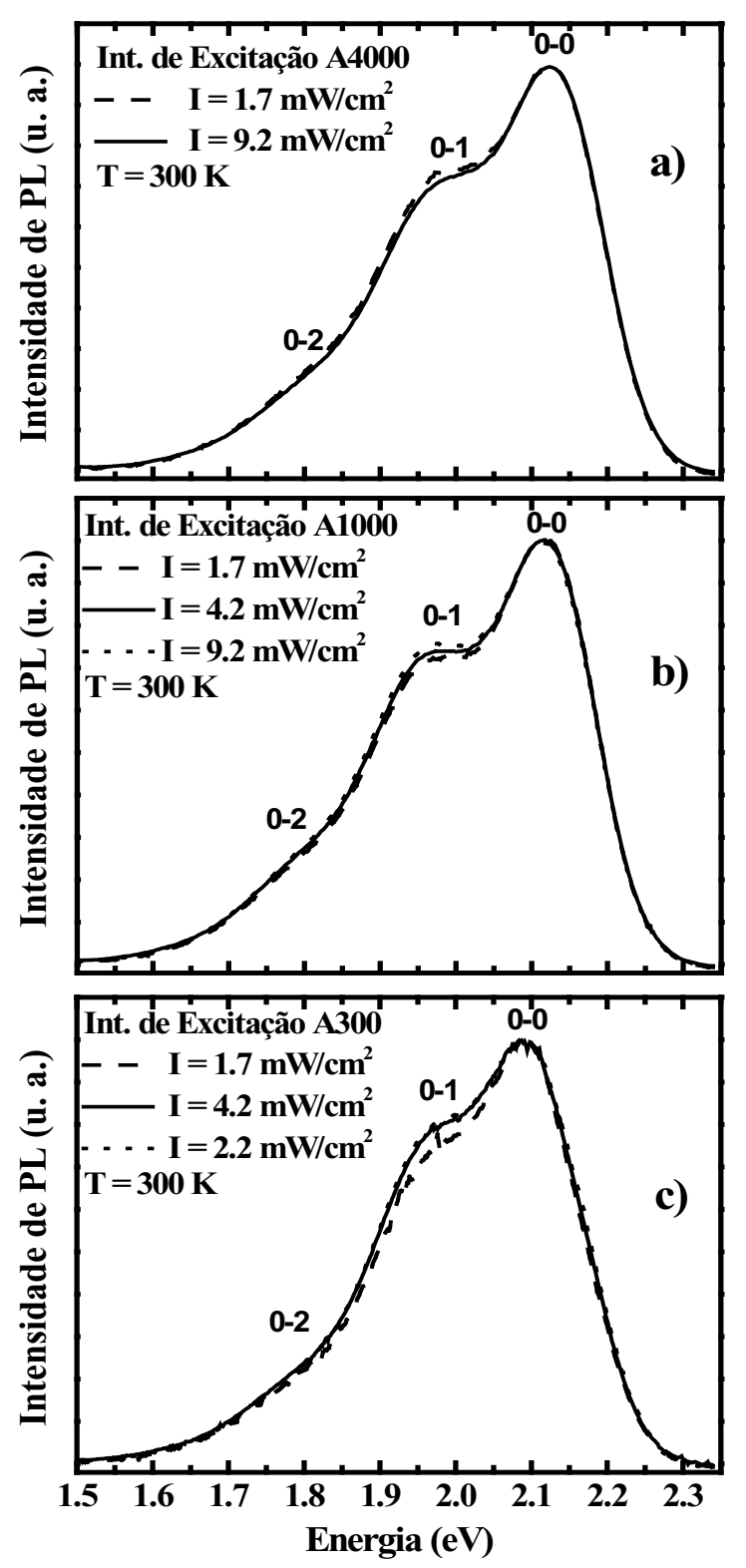

Figura 15. Espectros de fotoluminescência para diferentes intensidades de excitação a temperatura ambiente $(\mathrm{T}=$ 300 K). a) amostra A4000, b) amostra A1000 e c) amostra A300.

Um estudo mais sistemático da fotoluminescência em função da temperatura é realizado na amostra A1000 preparada com rotação intermediária. Apresentamos na Figura 16 as medidas de fotoluminescência realizadas nessa amostra no intervalo de temperatura de $130 \mathrm{~K}$ a $290 \mathrm{~K}$ sob mesma intensidade de excitação $\left(1.7 \mathrm{~mW} / \mathrm{cm}^{2}\right)$.

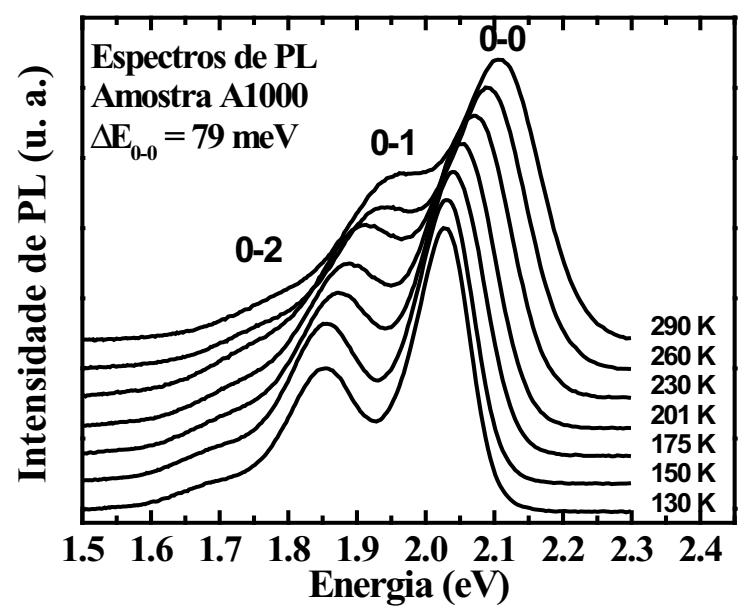

Figura 16. Espectros de PL para todas as temperaturas.

O máximo das transições (0-0), (0-1) e (0-2), medidos a $\mathrm{T}=130 \mathrm{~K}$, têm energia de $2.029 \mathrm{eV}$, $1.85 \mathrm{eV}$ e $1.68 \mathrm{eV}$, respectivamente. Podemos observar um "blue-shift" do máximo dos picos com o aumento da temperatura característico dos polímeros conjugados.

O ajuste dos espectros de fotoluminescência foi realizado com o emprego de gaussianas, de acordo com o procedimento efetuado por Zeng e Ding (2004) e Wantz et al. (2005) para ajustar espectros de fotoluminescência do PPV e Kong et al. (2005) para ajustes em espectros de PL de nano-MEHPPV “arrays". Na Figura 17 mostramos o ajuste dos espectros obtidos a $130 \mathrm{~K}, 201 \mathrm{~K}$ e $290 \mathrm{~K}$.

Os ajustes com gaussianas, entretanto apresentam problemas. Pequenas variações no procedimento podem levar a resultados pouco claros que prejudicam a análise do comportamento óptico do filme. Para mostrar isto, desenvolvemos aqui dois procedimentos:

i) ajuste com três gaussianas sem parâmetros fixos. O programa de ajuste através de cálculos de mínimos quadrados encontra os melhores parâmetros para ajustar as três gaussianas com os dados experimentais. Apresentamos os resultados dos ajustes para três temperaturas $(130 \mathrm{~K}, 201 \mathrm{~K} \mathrm{e}$ 290 K) na Figura 17. 
ii) ajuste com três gaussianas com dois parâmetros fixos. Fixamos a pico de energia e a intensidade das gaussianas 1 e 2 com o máximo da transição (0-0) e (0-1). A terceira gaussiana, que corresponde ao ombro no lado de menor energia, é trabalhada com os parâmetros livres. Apresentamos os resultados dos ajustes para três temperaturas $(130 \mathrm{~K}, 201 \mathrm{~K}$ e 290 K) na Figura 18.

Os máximos dos espectros de PL, na Figura 18 , não necessariamente precisam coincidir com a curva resultante da soma das três gaussianas, pois a soma das caudas das gaussianas influencia muito a forma final. Além disso, a largura de linha a meia altura FWHM ("Full Width Half Maximum") da banda eletrônica não deve ser a mesma quando há interação do elétron com um modo vibracional. Isto é, não há conservação de energia, pois os elétrons decaem radiativamente e sofrem processos de relaxação diversos e estes processos devem estar agindo nas bandas (0-1) e (0-2). Entretanto, as bandas vibrônicas correspondem a diferentes modos de vibração. Temos pelo menos quatro modos vibracionais para o MEH-PPV, conforme se pode verificar no espectro Raman (Figura 10) com energias na região correspondente a banda vibrônica (0-1). Um método de ajuste considerando os diferentes modos vibracionais, recentemente desenvolvido por Cury et al. (2004), foi aplicado ao BEH-PPV e mostrou que com o aumento da temperatura os diferentes modos podem perder ou ganhar intensidade, o que interfere na forma de linha do espectro de fotoluminescência de modo decisivo. Contudo, este método foi utilizado com o parâmetro FWHM fixo e não fornece um bom ajuste da forma de linha, embora permita uma análise mais sistemática dos processos físicos envolvidos na emissão de luz pelo material.

O que observamos aqui é que usando o procedimento $i$ ) (Figura 17) conseguimos um ótimo ajuste, porém, apesar do programa ajustar a curva experimental por meio do método de mínimos quadrados, não obtivemos resultados físicos coerentes e discutiremos esses fato na análise do parâmetro de Huang-Rhys mais a frente (ver Figura 22). Assim, optamos aqui por centrar as gaussianas 1 e 2 no máximo das transições (0-0) e (0-1) que são bem definidas no intervalo de temperatura analisado, deixando solta a gaussiana 3 , que está associada ao ombro que não fica bem definido em temperatura alguma. Com isso, perdemos um pouco no ajuste da forma de linha, mas ganhamos uma melhor compreensão dos processos físicos existentes no material.

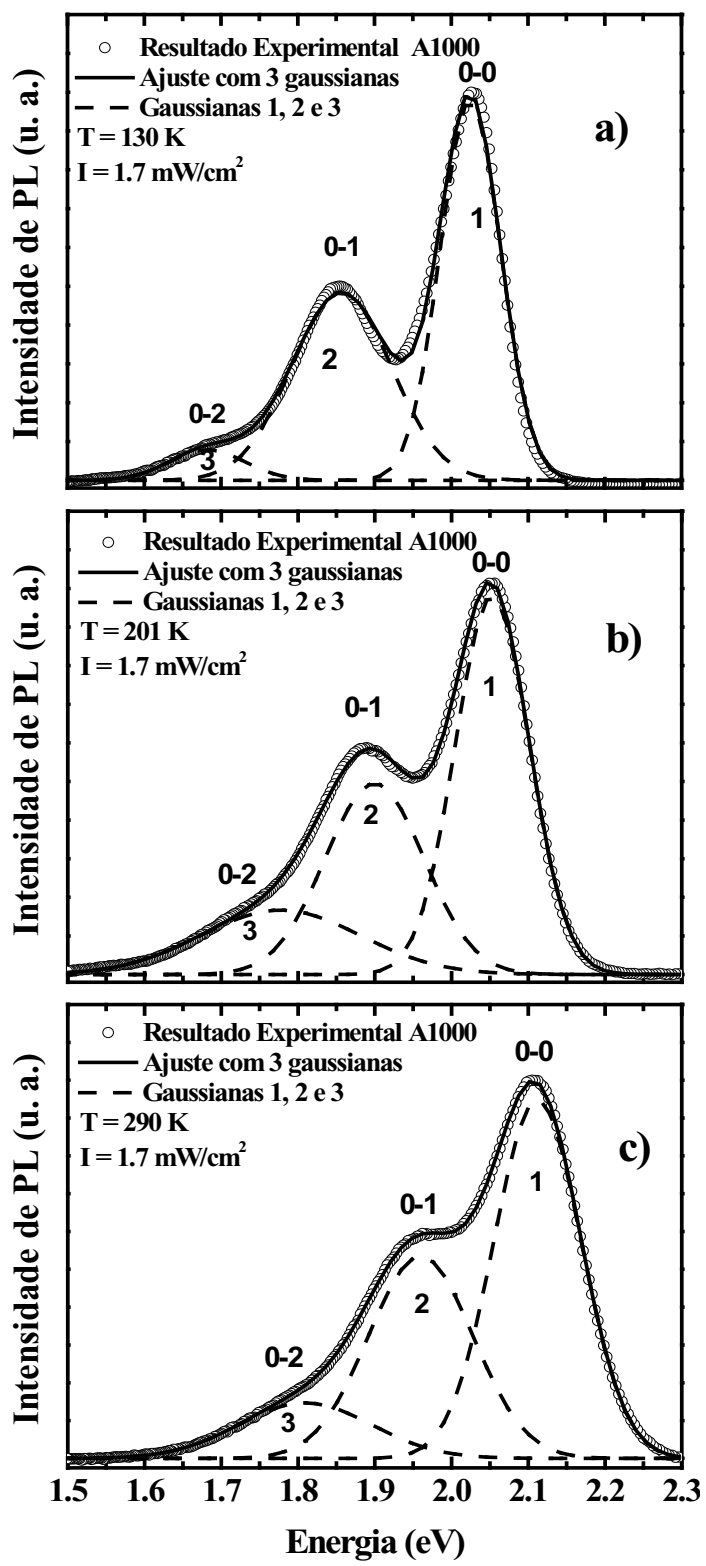

Figura 17. Espectros de fotoluminescência nas temperaturas de $130 \mathrm{~K}, 201 \mathrm{~K}$ e $290 \mathrm{~K}$ e intensidade de excitação de $1.7 \mathrm{~mW} / \mathrm{cm}^{2}$ ajustados com três gaussianas sem parâmetros fixos. 

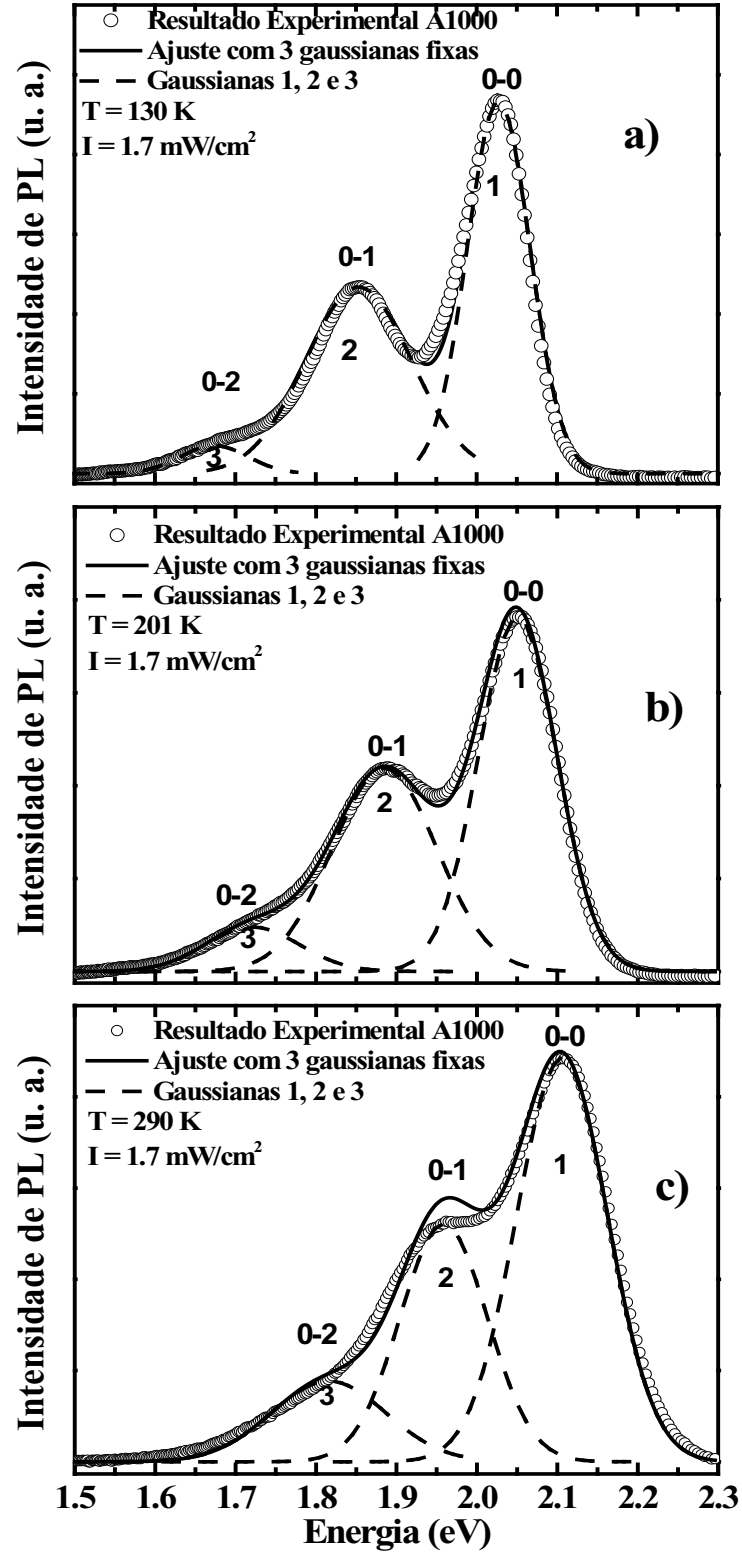

Figura 18. Espectros de fotoluminescência nas temperaturas de $130 \mathrm{~K}, 201 \mathrm{~K}$ e $290 \mathrm{~K}$ e intensidade de excitação de $1.7 \mathrm{~mW} / \mathrm{cm}^{2}$ ajustados com três gaussianas com dois parâmetros fixos.

A variação do pico de energia da transição puramente eletrônica (0-0) com a temperatura e o ajuste com a Equação 2.17 são mostrados na Figura 19. Mostramos ainda, nesta figura, a variação do pico (0-1) com a temperatura.

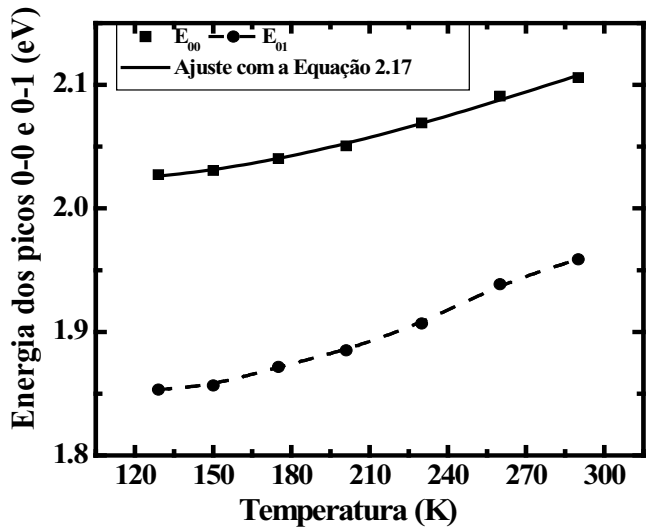

Figura 19. Variação da posição do pico (0-0), quadrado, do pico (0-1), círculo cheio com a temperatura. A linha cheia é o ajuste obtido com a Equação 2.17 para os dados experimentais da posição do pico (0-0). A linha tracejada é apenas um guia para os olhos.

O deslocamento da transição puramente eletrônica e da primeira banda vibrônica no intervalo de temperatura analisado é de $86 \mathrm{meV}$ e $105 \mathrm{meV}$, respectivamente. O "blue-shift" do pico (0-0), induzido pelo aumento da temperatura, foi observado também no PPV e seus derivados em estudos de fotoluminescência. Yu et al. (1996) e Hagler et al. (1991) observaram no PPV uma variação de aproximadamente $55 \mathrm{meV}$ e $60 \mathrm{meV}$ nos intervalos de temperatura de 77 a $300 \mathrm{~K}$ e 80 a $300 \mathrm{~K}$, respectivamente. Oliveira et al. (2003) encontraram um deslocamento de aproximadamente $50 \mathrm{meV}$ para o BEH-PPV com a temperatura variando de 15 a $294 \mathrm{~K}$. Quan et al. (2006) mediram um "blue-shift" de $60 \mathrm{meV}$ para o pico (0-0) e de $120 \mathrm{meV}$ para o pico (0-1) no MEH-PPV no intervalo de temperatura de 77 a 294 K. Estes autores interpretaram o fenômeno como resultado da diminuição do comprimento de conjugação efetivo devido aos movimentos torcionais que aumentam quando a temperatura aumenta. Tal efeito reduz a delocalização dos elétrons $\pi$ levando a um maior confinamento e, portanto, a um aumento da energia de transição. 
O deslocamento da transição puramente eletrônica está de acordo com aquele encontrado por outros autores (PICHLER et al., 1993; YALIRIKI; SILBEY, 1996) eé previsto pela Equação 2.17 através da curva contínua na Figura 19. Do ajuste, a energia que ativa o processo de quebra de conjugação obtida é $\varepsilon=57.1 \mathrm{meV}$. Este valor é maior que o encontrado para o BEH-PPV $(\varepsilon=28 \mathrm{meV})$. Essa diferença pode, entretanto, estar relacionada ao restrito intervalo de temperatura em que nosso experimento foi realizado além das diferenças estruturais entre os polímeros.

O valor de $\mathrm{E}_{0}$ (o "gap" de energia extrapolado para $\mathrm{T}=0 \mathrm{~K}$ ) é de $2.021 \mathrm{eV}$, próximo do valor encontrado na literatura para MEH-PPV/ITO (QUAN et al., 2006). $\mathrm{O}$ valor de $\Delta \mathrm{E}$ (variação máxima de energia da linha (0-0) no processo de emissão) encontrado é $0.85 \mathrm{eV}$, bastante superior à encontrada para o PPV que é de $0.40 \mathrm{eV}$ (BORGES, 2001) e para o BEHPPV que é de 0.12 eV (OLIVEIRA, 2004). Essa grande diferença pode estar relacionada ao intervalo de temperatura relativamente restrito de nossas medidas, qualidade da amostra e/ ou procedimento de ajuste.

A energia de separação dos picos (0-0) e (0-1), ou (0-1) e (0-2) obtida neste trabalho depende mais fortemente da temperatura do que a obtida por Wantz et al. (2005). Naquele trabalho eles realizaram medidas de eletroluminescência no intervalo de 80 a $350 \mathrm{~K}$ no MEH-PPV e obtiveram uma separação em energia dos picos que não variou tanto com a temperatura, entretanto foram apresentados os dados experimentais para que pudéssemos fazer uma melhor comparação.

Na Figura 20, apresentamos a dependência da intensidade do pico (0-0) com a temperatura. A linha cheia é o ajuste dos dados experimentais com a Equação 2.18.

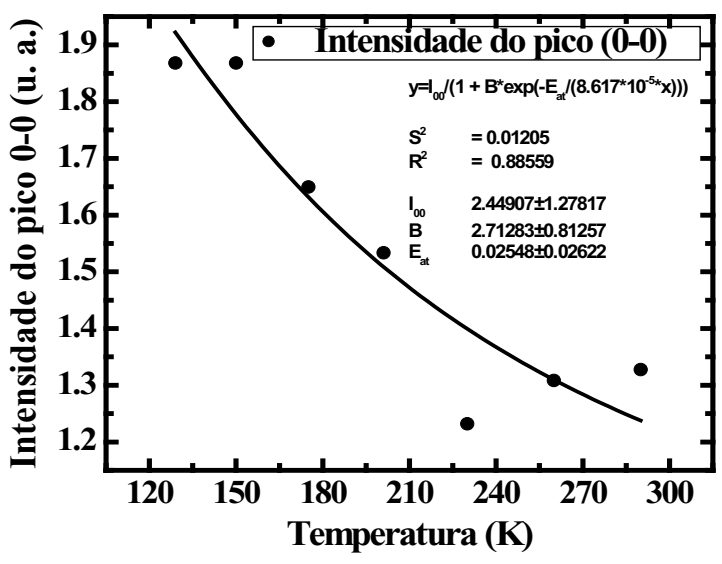

Figura 20. Dependência da intensidade do pico (0-0) com a temperatura. A linha cheia é o ajuste com a Equação 2.18 .

O aumento da intensidade da linha (0-0) mostra que, com o decréscimo da temperatura, existe uma supressão de vias não radiativas de relaxamento para o elétron excitado (ou éxciton). Quanto maior a temperatura, maior será a probabilidade de espalhamento por modos vibracionais da molécula. Assim, espera-se que a probabilidade de ocorrer a transição puramente eletrônica (pico (0-0) nos espectros de PL) decresça, o que leva a uma diminuição na intensidade da linha $\mathrm{I}_{00}$. A redução da eficiência da emissão é uma conseqüência da ativação de canais não radiativos, provavelmente devido a processos multifônons. Essa redução é descrita pela expressão 2.18. Por meio dessa equação, ajustamos a curva experimental e obtivemos uma energia de ativação dos processos de decaimento não radiativo $\left(\mathrm{E}_{\mathrm{at}}\right)$ de $25.5 \mathrm{meV}$. Essa energia corresponde à altura da barreira de potencial que o elétron tem que vencer quando ativado termicamente no estado excitado para então decair não radiativamente através dos níveis vibracionais do estado fundamental. A altura da barreira $E_{a t}$ é proporcional ao valor do acoplamento elétron-fônon medido pelo fator Huang-Rhys S. O valor de $\mathrm{E}_{\mathrm{at}}$ encontrado para o BEH-PPV é $30 \mathrm{meV}$ (OLIVEIRA, 2004). 
Na Figura 21, apresentamos a dependência da largura de linha a meia altura (FWHM) do pico de transição puramente eletrônico (0-0) com a temperatura obtidas. Os dados foram obtidas pelo emprego dos diferentes procedimentos de ajuste com as gaussianas sem parâmetros fixos (círculos cheios) e com as gaussianas com a intensidade e a posição fixos (quadrados vazios).

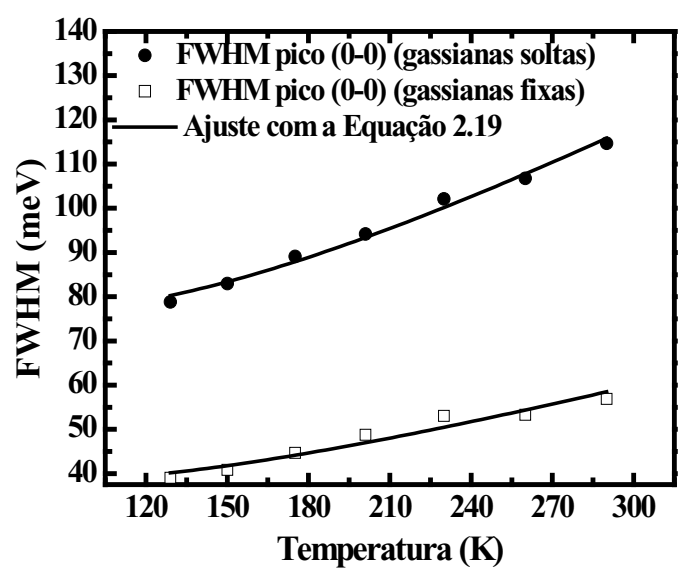

Figura 21. Variação da largura de linha a meia altura do pico (0-0) com a temperatura obtida pelos dois procedimentos de ajuste com gaussianas soltas (círculo cheio) e com gaussianas fixas (quadrado vazio). A linha contínua representa o ajuste com a Equação 2.19.

A FWHM(T) aumenta com a temperatura, de acordo com os processos de desordem térmica (alargamento homogêneo) e este alargamento pode ser bem descrito pela Equação 2.19-linhas contínuas de Figura 21 - em toda a faixa de temperatura medida, para ambos os procedimentos de ajuste. $\mathrm{O}$ valor do alargamento não- homogêneo $\left(\Gamma_{0}\right)$ dobrou de um ajuste para o outro, de $37.6 \mathrm{meV}$ (gaussianas fixas) para $75.6 \mathrm{meV}$ (gaussianas livres). A energia efetiva de fônon () variou muito pouco de 38.2 $\mathrm{meV}$ (gaussianas fixas) para $39 \mathrm{meV}$ (gaussianas livres). Essa energia corresponde à energia térmica necessária para ativar mudanças conformacionais na cadeia polimérica.
A FWHM aumenta de $78.8 \mathrm{meV}$ com o ajuste por gaussianas livres (39 meV para gaussianas fixas) em $130 \mathrm{~K}$ para $113.6 \mathrm{meV}$ (56.7 meV para gaussianas fixas) em $290 \mathrm{~K}$. Obtém-se, assim, uma variação de $34.8 \mathrm{meV}$ com gaussianas livres $(17.7 \mathrm{meV}$ para gaussianas fixas). Essa diferença mostra como os procedimentos de ajuste podem influenciar na análise dos resultados experimentais. Vários resultados na literatura mostram uma relativa dispersão de valores do parâmetro FWHM(T). Borges (2001) obteve um alargamento de $31 \mathrm{meV}$ no intervalo de $40 \mathrm{~K}<\mathrm{T}<$ $180 \mathrm{~K}$, e enquanto Zeng e Ding (2004) obtiveram um alargamento de $140 \mathrm{meV}$ para uma variação de temperatura de 83 a $294 \mathrm{~K}$, ambos no PPV. Oliveira (2003) obteve uma aumento de $45 \mathrm{meV}$ na faixa de temperatura de 15 a $297 \mathrm{~K}$ no BEH-PPV. Quan et al. (2006) obtiveram um alargamento de linha de 105 meV para T, variando entre 77 e $294 \mathrm{~K}$ no $\mathrm{MEH}-$ PPV, e em medidas de eletroluminescência Wantz et al. (2005), também no MEH-PPV (no intervalo de temperatura de 80 a $350 \mathrm{~K}$ ) observaram um alargamento de $80 \mathrm{meV}$. É possível, assim, que além dos fatores associados à preparação (solvente, velocidade de rotação no caso da técnica de "spincoating”) e as diferentes técnicas de preparação empregadas, a dispersão de valores esteja também associada ao procedimento de ajuste da forma de linha empregado por cada autor.

Na Figura 22, mostramos variação do fator de Huang-Rhys com a temperatura usando os dois procedimentos citados anteriormente. 


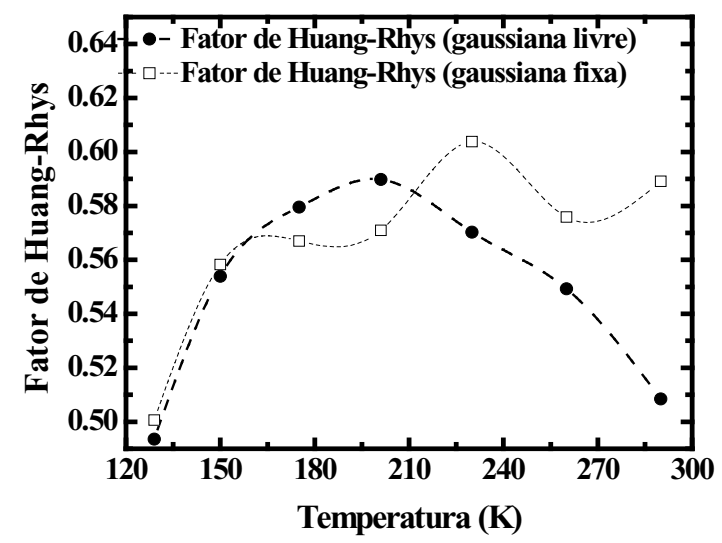

Figura 22. Variação do fator Huang-Rhys com a temperatura. O círculo cheio representa o fator HuangRhys com $\mathrm{I}_{01}$ e $\mathrm{I}_{00}$ obtidos pelo ajuste com gaussianas soltas e o quadrado vazio representa o fator Huang-Rhys com $I_{01}$ e $I_{00}$ obtidos com gaussianas fixas. As linhas tracejadas são guias para os olhos.

O fator de Huang-Rhys utiliza as intensidades $\mathrm{I}_{01}$ e $\mathrm{I}_{00}$ ambas obtidas dos ajustes com gaussianas que tiveram a intensidade e posição em energia fixas nos valores dos máximos das transições (0-1) e (0-0). Esse fator está mais coerente com o encontrado na literatura (QUAN et al., 2006) do que o obtido com os parâmetros do ajuste com três gaussianas sem parâmetros fixos. A Equação 2.20 não ajustou os dados experimentais da Figura 22. No entanto, é importante salientar que este procedimento para calcular $\mathbf{S}\left(\mathbf{S}=\mathrm{I}_{01} / \mathrm{I}_{00}\right)$ é utilizado quando se tem apenas um modo vibracional isolado o que, para o caso do MEH-PPV, deve ser considerado como uma primeira aproximação.

O comprimento de conjugação pode ser encontrado através da comparação do fator de Huang-Rhys coma Equação 2.20, mas não obtivemos ajuste empregando esta equação. Outra maneira de se obter o comprimento de conjugação $\mathbf{n}$ é utilizar a Equação 2.21 com os valores empíricos de $a$ e $b$, iguais a 3.2 e 38 , respectivamente. A variação do comprimento de conjugação, encontrado por meio da Equação 2.21, é apresentado na Figura 23.

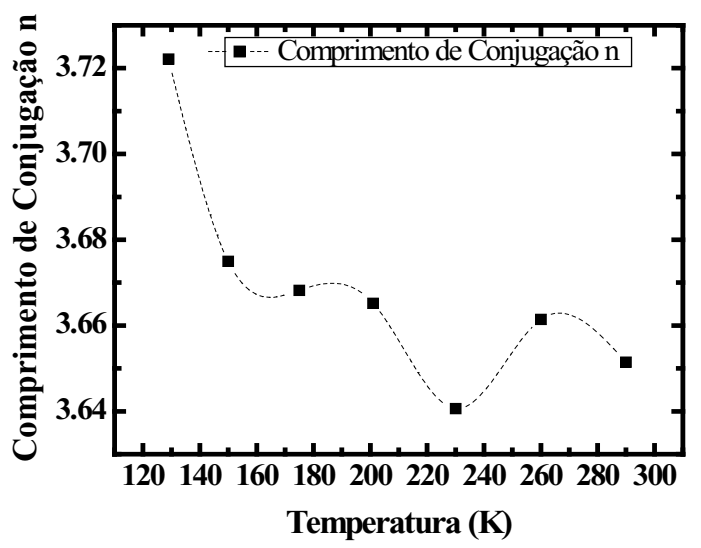

Figura 23. Dependência do comprimento de conjugação calculado pela Equação $2.21 \mathrm{com}$ a temperatura. A linha tracejada é um guia para os olhos.

O comprimento de conjugação ficou bem abaixo do encontrado na literatura para o PPV (ZENG; DING, 2004), $\mathrm{n}=7$, utilizando a Equação $2.21 \mathrm{e}$ para o BEH-PPV (OLIVEIRA et al., 2003), $\mathrm{n}_{0}=13$, utilizando a Equação 2.20. Esta grande diferença em relação aos nossos dados está relacionada ao intervalo relativamente pequeno de temperatura analisado em nosso trabalho, com limite inferior em uma temperatura relativamente alta $(130 \mathrm{~K})$. É possível que medidas em um intervalo maior de temperatura, no sentido de temperaturas mais baixas, permitam-nos obter valores mais adequados para o comprimento de conjugação.

\section{Conclusão}

Apresentamos um estudo sistemático da dependência com a temperatura dos espectros de fotoluminescência do polímero conjugado $\mathrm{MEH}$ PPV preparados pela técnica de "spin-coating". Nossos resultados apresentam um "blue-shift" da energia de transição de PL dos picos (0-0), (0-1) e (0-2) com o aumento da temperatura, típico dos polímeros conjugados, que é explicado pelo aumento do comprimento de conjugação efetivo (n) com a diminuição da temperatura do filme, devido à supressão da desordem térmica. 
A comparação da homogeneidade dos filmes com a velocidade de rotação em que foram preparados nos confirmou que filmes mais homogêneos são aqueles preparados em maior velocidade de rotação.

Em baixas temperaturas, os espectros de PL da amostra A1000 (1000 rpm) mostraram em pico estreito para transição puramente eletrônica e uma série de bandas vibrônicas que revelam o acoplamento com diferentes modos vibrônicos.

A dependência dos parâmetros (energia, largura de linha, intensidade) do pico (0-0) com a temperatura e seus ajustes nos deram uma boa visão do comportamento físico da amostra analisada.

O uso de gaussianas no ajuste dos espectros de fotoluminescência, apesar de levar a um ótimo ajuste, não possibilita uma análise adequada dos parâmetros que envolvem a intensidade dos picos e os modos vibrônicos do polímero (como o fator Huang-Rhys). Um modelo de ajuste que considera os modos vibrônicos que compõem a banda vibrônica (0-1) foi proposto recentemente por Cury et al. (2004) e será utilizado em um trabalho futuro, no qual estudaremos a fotoluminescência em função da temperatura em um conjunto maior de amostras.

\section{Referências}

BORGES, C. A. M. Processos radiativos e não radiativos em polímeros conjugados emissores de luz. 2001. Dissertação. (Mestrado em Física) - Universidade de São Paulo, São Carlos.

BRAUN, D.; HEEGER, A. J. Visible light emission from semiconducting polymer diodes. Applied Physics Letters, New York, v. 58, n. 18, p. 1982-1984, 1991.

BRAZOVSKII, S.; KIROVA, N.; BISHOP, A. R. Theory of electronic states and excitations in PPV. Optical Material, Lyon, v. 9, n. 1-4, p. 465-471, 1998.

BURROUGHES, J. H.; BRADLEY, D. D. C.; BROWN, A. R.; MARKS, R. N.; MACKAY, K.; FRIEND, R. H.; BURN, P. L.; HOLMES, A. B. Light-emitting diodes based on conjugated polymers. Nature, London, v. 347, n. 6293 , p. $539-541,1990$.

CANEVAROLO JÚNIOR., S. V. Ciência dos Polímeros. São Paulo: Artliber, 2002.
CHEN, S.; MENG, H. F. Intrachain carrier transport in conjugated polymer with structural and chemical defects. Physical Review B, New York, v. 66, n. 3, p. 035202.1035202.5, 2002.

CHIANG, C. K.; FINCHER JÚNIOR., C. R.; PARK, Y. W.; HEEGER, A. J.; SHIRAKAWA, H. E.; LOUIS, E. J. Electrical conductivity in doped polyacetylene. Physical Review Letter, New York, v. 39, n. 17, p. 1098-1101, 1977.

CÔTÉ, M. Electronic properties of polymers: a microscopic view. Physics in Canada, Ottawa, v. 59, n. 2, p. 1-15, 2003

CURY, L. A. Absorção e fotoluminescência em polímeros conjugados. Belo Horizonte: Ed. da UFMG, 2004. Comunicação Interna DF/ICEX.

CURY, L. A.; GUIMARÃES, P. S. S.; MOREIRA, R. L.; CHACHAM, H. Asymmetric line shape in the emission spectra of conjugated polymer thin films: An experimental signature of one-dimensional electronic states. Journal Chemical Physics, New York, v. 121, n. 8, p. 3836-3839, 2004.

FRIEND, R. H.; BRADLEY, D. D. C.; TOWNSEND, P. D. Photo-excitation in conjugated polymers. Journal Physics.: Applied Physics, New York, v. 20, n. 11, p. 1367-1384, 1987.

GETTINGER, C. L.; HEEGER, A. J.; DRAKE, J. M.; PINE, D. J. A photoluminescence study of poly(phenylene vinylene) derivatives: The effect of intrinsic persistence length. Journal Chemical Physics, New York, v. 101, n. 2, p. 1673-1678, 1994.

GREENHAM, N. C.; FRIEND, R. H. Quantum theory of solids: semiconductor physics of conjugated polymer. Cambridge: Oxford at the Clarendon Press, 1955.

GULLET, J. Polymer photophysics and photochemistry. Cambridge: Cambridge University Press, 1987.

HAGLER, T. W.; PAKBAZ, K.; VOSS, K. F.; HEEGER, A. J. Enhanced order and electronic delocalization in conjugated polymers oriented by gel processing in polyethylene. Physical Review B, New York, v. 44, n. 16, p. 8652-8666, 1991.

HEEGER, A. J. Nobel Lecture: semiconducting and metallic polymers: the fourth generation of polymeric materials. Reviews of Modern Physics, New York, v. 73, n. 3, p. 681-700, 2001.

HENLEY, R.; KOHLER, E. B. Electronic structure of polyenes related to the visual chromophore. A simple model for the observed band shapes. Biophysical Journal, Standford, v. 20, n. 3, p. 377-382, 1977. 
KEIL, T. H. Shapes of Impurity Absorption Bands in Solids. Physical Review A, New York, v. 140, n. 2A, p. 601-617, 1965.

KIROVA, N.; BRAZOVSKII, S.; BISHOP, A. R. A systematic theory for optical properties of phenylenebased polymers. Synthetic Metals, Columbus, v. 100, n. 1, p. 29-53, 1999.

KIVELSON, S.; SHRIEFFLER, J. R.; HEEGER, A. J.; SU, W. P. Solitons in conducting polymers. Reviews of Modern Physics, New York, v. 60, n. 3, p. 781-850, 1988 .

KONG, F.; WUA, X. L.; HUANG, G. S.; YANG, Y. M.; YUAN, R. K.; YANG, C. Z.; CHU, P. K.; SIU, G. G. Optical emission from nano-poly[2-methoxy-5-(2'ethyl-hexyloxy)-p-phenylene vinylene] arrays. Journal Applied Physics, New York, v. 98, n. 7, p. 074304-1074304-4, 2005.

KRANZELBINDER, G.; LEISING, G. Organic solidstate lasers. Reports on Progress in Physics, Bristol, v. 63, n. 5, p. 729-762, 2000.

LIN, S. H. Band Shape of the Circular Dichroism. Journal Chemical Physics, New York, v. 55, n. 7, p. 3546-3554, 1971.

Effect of high pressures on molecular electronic spectra and electronic relaxation. Journal Chemical Physics, New York, v. 59, n. 8, p. 4458-4467, 1973.

LOURENÇO, S. A.; DIAS, I. F. L; DUARTE, J. L.; LAURETO, E; POÇAS, L. C.; TOGINHO FILHO, D. O.; LEITE, J. R. Thermal Espansion Contribution to the Temperature Dependence of Excitonic Transition in GaAs and AlGaAs. Brazilian Journal of Physics, São Paulo, v. 34, n. 2A, p. 517-525, 2004.

MARLETTA et al., 2001.

MILLER, E. K.; YANG, C. Y.; HEEGER, A. J. Polarized ultraviolet absorption by a highly oriented dialkyl derivative of poly(paraphenylene vinylene). Physical Review B, New York, v. 62, n. 11, p. 6889-6891, 2000.

MILLER, E. K.; YOSHIDA, D.; YANG, C. Y.; HEEGER, A. J. Polarized ultraviolet absorption of highly oriented poly(2-methoxy, 5-(2'-ethyl)-hexyloxy) paraphenylene vinylene. Physical Review B, New York, v. 59, n. 7, p. 4661-4664, 1999.

MOSES, D.; FELDBLUM, A.; EHRENFREUND, E.; HEEGER, A. J.; CHUNG, T. C.; MACDIARMID, A. G. Pressure dependence of the photoabsorption of polyacetylene. Physical Review B, New York, v. 26, n. 6, p. 3361-3369, 1982.
NABETANI, A.; TOMIOKA, A.; TAMURU, H.; MIYANO, K. Optical properties of two-dimensional dye aggregate. Journal Chemical Physics, New York, v. 102, p. 5109-5117, 1995.

OLIVEIRA, F. A. C. Efeito da temperatura nas propriedades vibrônicas dos polímeros conjugados. 2004. Dissertação (Mestrado em Física) - Universidade Federal de Minas Gerais, Belo Horizonte.

OLIVEIRA, F. A. C.; CURY, L. A.; RIGHI, A.; MOREIRA, R.L.; GUIMARAES, P. S. S.; MATINAGA, F. M.; PIMENTA, M. A.; NOGUEIRA, R. A. Temperature effects on the vibronic spectra of BEH-PPV conjugated polymer films. Journal Chemical Physics, New York, v. 119, n. 18, p. 9777-9782, 2003.

PARKER, I. D. Carrier tunneling and device characteristics in polymer light-emitting diodes. Journal Applied Physics, New York, v. 75, n. 3, p. 1656-1666, 1994.

PEETERS, E.; RAMOS, A. M.; MESKERS, S. C. J.; JANSSEN, R. A. J. Singlet and triplet excitations of chiral dialkoxy-p-phenylene vinylene oligomers. Journal Chemical Physics, New York, v. 112, n. 21, p. 9445-9454, 2000 .

PEIERLS, S. R. Quantum theory of solids. London: Oxford University Press, 1995.

PHILLIPS, D. Polymer photophysics, luminescence, energy migration and molecular motion in synthetic polymers. Cambridge: Cambridge University Press, 1985.

PICHLER, K.; HALLIDAY, D. A.; BRADLEY, D. D. C.; BURN, P. L.; FRIEND, R. H.; HOLMES, A. B. Optical spectroscopy of highly ordered poly(p-phenylene vinylene). Journal Physics: Condensed Matter, London, v. 5, n. 38, p. 7155-7172, 1993.

QUAN, S.; TENG, F.; XU, Z.; ZHANG, T.; QIAN, L.; LIU, D.; HOU, Y.; WANG, Y. Temperature effects on photoluminescence of poly[2-methoxy-5-(20-ethylhexyloxy)-1,4-phenylene vinylene]. Material Letter, Amsterdam, v. 60, n. 9-10, p. 1134-1136, 2006.

SCOTT, J. C.; KAUFMAN, J. H.; BROCK, P. J.; DiPIETRO, R.; SALEM, J.; GOITIA, J. A. Degradation and failure of MEH-PPV light-emitting diodes. Journal Applied Physics, New York, v. 79, n. 5, p. 2745-2751, 1996. 
SHIRAKAWA, H. E.; LOUIS, E. J.; MACDIARMID, A. G.; CHIANH, C. K.; HEEGER, A. J. Synthesis of electrically conducting organic polymers - halogen derivatives of polyacetylene, $(\mathrm{CH})$. Journal of the Chemical Society: Chemical Communtication, New York, v.16, p. 578-580, 1977.

SILVA, M. A. T.; DIAS, I. F. L; DUARTE, J. L.; LAURETO, E; SILVESTRE, I.; CURY, L. A. GUIMARÃES, P. S. S. Identification of the optically active vibrational modes in the photoluminescence of MEH-PPV films. Journal Chemical Physics, New York, v. 128, n. 9, p. 094902-1-094902-7, 2008.

SU, W. P.; SCHRIEFFER, J. R.; HEEGER, A. J. Soliton excitations in polyacetylene. Physical Review B, New York, v. 22, n. 4, p. 2099-2111, 1980.

Solitons in Polyacetylene. Physical Review Letter, New York, v. 42, n. 25, p. 1698-1701, 1979.

TESSLER, N.; DENTON, G. J.; FRIEND, R. H. Lasing from conjugated-polymer microcavities. Nature, London, v. 382, n. 6593, p. 695-697, 1996.

WANTZ, G.; HIRGH, L.; HUBY, N.; VIGNAL, L.; BARRIÈRE, A. S.; PARNEIX, J. P. Temperaturedependentelectroluminescencespectraofpoly(phenylenevinylene) derivatives-based polymer light-emitting diodes. Journal Applied Physics, New York, v. 97, n. 3, p. 34505, 2005.
WOO, H. S.; LHOST, O.; GRAHAM, S. C.; BRADLEY, D.D.C.; FRIEND, R.H.; QUATTROCCHI, C.;BREDAS, J. L.; SCHENK, R.; MULLEN, K. Optical spectra and excitations in phenylene vinylene oligomers. Synthetic Metals, Columbus, v. 59, n. 1, p. 13-28, 1993.

YALIRAKI, S. N.; SILBEY, R. J. Conformational disorder of conjugated polymers: implications for optical properties. Journal Chemical Physics, New York, v. 104, n. 4, p. 1245-1253, 1996.

YANG, X.; MO, Y.; YANG, W.; YU, G.; CAO, Y. Efficient polymer light emitting diodes with metal fluoride/Al cathodes. Applied Physics Letters, New York, v. 79, n. 5, p. 563-565, 2001.

YU, J.; HAYASHI, M.; LIN, S. H.; FANN, W. S.; LIANG, K. K.; HSU, J. H.; CHAO, C. I.; CHUANG, K. R.; CHEN, S. A. Temperature effect on the electronic spectra of poly(p-phenylenevinylene). Synthetic Metals, Columbus, v. 82, n. 2, p. 159-166, 1996.

ZENG, Q. G.; DING, Z. J. Photoluminescence and Raman spectra study of para-phenylenevinylene at low temperatures. Journal Physics Condensed Matter, London, v. 16, n. 28, p. 5171-5178, 2004. 